\title{
Belphégor
}

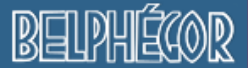

Littérature populaire et culture médiatique

17 | 2019

Mutations des légitimités dans les productions

culturelles contemporaines

\section{Partager son sujet et son expérience de recherche dans un carnet de thèse : des doctorant.es entre recherche de légitimité et recherche de sens}

\section{Mélodie Faury}

\section{(2) OpenEdition}

\section{Journals}

Electronic version

URL: https://journals.openedition.org/belphegor/1779

DOI: 10.4000/belphegor.1779

ISSN: 1499-7185

Publisher

LPCM

Electronic reference

Mélodie Faury, "Partager son sujet et son expérience de recherche dans un carnet de thèse : des doctorant.es entre recherche de légitimité et recherche de sens", Belphégor [Online], 17 | 2019, Online since 27 March 2019, connection on 25 August 2021. URL: http://journals.openedition.org/belphegor/ 1779 ; DOI: https://doi.org/10.4000/belphegor.1779

This text was automatically generated on 25 August 2021

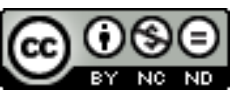

Belphégor est mis à disposition selon les termes de la Licence Creative Commons Attribution - Pas d'Utilisation Commerciale - Pas de Modification 4.0 International. 


\title{
Partager son sujet et son expérience de recherche dans un carnet de thèse : des doctorant.es entre recherche de légitimité et recherche de sens
}

\author{
Mélodie Faury
}

1 Ancrée dans les études de sciences et les sciences de l'information et de la communication, je m'intéresse aux pratiques de communication des doctorant.es depuis ma thèse, selon une approche communicationnelle fondée sur un empirisme épistémologique et sur la réflexivité comme posture discursive (Faury, 2012; Le Marec, 2002).

2 J'ai précédemment démontré comment ces pratiques de communication des doctorant.es, prises en tant qu'objets de recherche sur la recherche, et les discours qu'elles et ils mobilisent rendent intelligibles leurs pratiques vécues de la recherche, c'est-à-dire leur expérience, permettant de parcourir un espace mental de la recherche et un rapport identitaire et culturel aux sciences (Faury, 2012). Je définis l'espace mental de la recherche comme un espace physique et symbolique que les doctorant.es investissent à partir de ce qu'elles et ils imaginent que la recherche est ou doit être («bon » chercheur, " bonnes " pratiques, idéal vers lequel tendre) et des conditions de réalisation effectives qu'elles et ils éprouvent (conditions matérielles, statut du doctorant, relations aux collègues du laboratoire et notamment avec le directeur ou la directrice de thèse). Dans ce sens, le carnet de thèse témoigne d'une certaine conception de la recherche et d'une recherche située dans une discipline, un laboratoire, un terrain, etc. Plus précisément, le carnet de thèse peut être considéré comme un espace numérique, à l'intersection entre les enjeux (normes, valeurs) de la recherche comme pratique professionnelle ancrée dans des institutions et ceux du blog, en tant que genre (Couleau et Hellégouarc'h, 2010). Cet espace est investi de diverses manières par les doctorant.es, 
selon la représentation et le sens qu'elles et ils donnent d'une part à leur statut de doctorant.e et d'autre part aux fonctions attribuées à un blog associé à une activité de recherche.

\section{Activité de doctorant.e, activité de carnetier.e et parcours de thèse : les pratiques de recherche et les écrits sur les pratiques}

\subsection{Carnets de thèse}

3 L'objet de l'étude est le "carnet de thèse ", en tant que blog individuel de jeunes chercheur.es en sciences humaines et sociales, hébergé sur la plateforme Hypotheses.org au sein du portail OpenEdition.

4 La plateforme Hypothèses a été créée en 2008. En septembre 2018, 2722 blogs sont publiés au catalogue sur environ 4200 blogs ouverts mais encore non répertoriés. On compte environ 20200 comptes utilisateurs sur Hypothèses, et 12,8 millions de visites en $2017^{1}$. On y trouve 17 catégories différentes de blog, appelées « types de carnets ${ }^{2}$ dont les « carnets de chercheurs", englobant 427 carnets au catalogue, et les "carnets de thèse » regroupant 175 blogs.

5 Je suis moi-même carnetière depuis ma thèse ${ }^{3}$, dans des carnets individuels et collectifs depuis 2010 ; je suis lectrice de nombreux carnets depuis la même date et je suis en conversation avec un certain nombre de carnetier.es, notamment via des carnets partagés, les commentaires et des discussions sur Twitter. Je développe une approche réflexive de ma pratique de carnetière, dans différents espaces et à partir de l'expérience vécue et située de l'activité. Enfin, je contribue au Conseil scientifique d'Hypotheses.org depuis 2011. Je travaille donc en "terrain familier", d'un point de vue situé de praticienne et d'un autre plus institutionnel et politique. À l'opposé de la posture de l'étranger mobilisée dans les travaux classique d'ethnographie de laboratoire (Latour et Woolgar, 1996), je choisis de prendre la familiarité avec le terrain de recherche pour point de départ, à la fois d'un questionnement de recherche et de la relation enquêteurenquêté que je tisse (Thiault, 2009 ; Faury, 2012). Mon rapport au terrain définit ma démarche épistémologique : je développe une mise en œuvre empirique de la réflexivité au moyen de l'approche communicationnelle et de situations d'hyperproximité (Schwartz, 2011 ; Le Marec, 2002 ; Faury, 2012).

6 L'approche qualitative que je présente s'intègre dans une recherche en cours, menée dans le cadre du projet HYCAR du GIS du Réseau des URFIST ${ }^{4}$, au sujet des carnets de doctorant.es et des carnets de chercheur.es de la plateforme Hypotheses.org. Elle est articulée avec des approches quantitatives d'analyse de données et la mise en œuvre d'entretiens exploratoires sur l'expérience vécue de l'activité de "carnetier.e »5. Dans cet article, je présente une analyse des carnets et des billets en tant que pratiques de communications au sein de l'activité de recherche, à la fois en tant qu'inscriptions matérielles et en tant que discours sur les pratiques ${ }^{6}$. Je prends les billets ${ }^{7}$ des carnets de thèse comme des unités d'analyse.

$7 \quad$ Les types de billets des carnets de thèse sont construits en prenant comme référentiel le parcours-type ${ }^{8}$ de la thèse. Il s'agit bien d'une typologie de la diversité des billets de « carnets de thèse » et non d'une répartition des formations discursives. Concrètement, 
les billets récoltés pour l'analyse sont choisis de la manière suivante : sélection des carnets de thèse via le catalogue d'OpenEdition, sélection des carnets francophones au sein de cette catégorie (151 carnets), récupération des billets récents présentés sur le catalogue pour l'ensemble de ces carnets (nombre variable selon les carnets : de trois à une dizaine).

\subsection{Parcours de doctorant.es}

8 Plusieurs arguments et hypothèses appuient le choix d'une étude qualitative des carnets de doctorant.es parmi l'ensemble des carnets de recherche.

En termes de parcours de recherche, la thèse est le moment du choix de s'inscrire ou non dans un métier, dans une discipline et un champ, dans un sujet de recherche, à partir notamment de l'expérience vécue et située de la pratique (Faury, 2012). Ce moment important se traduit par un questionnement explicite sur le sens d'une activité professionnelle. L'explicitation et la réflexivité sur l'expérience sont déployées notamment, par l'oral, dans les situations d'entretiens menées pour la recherche (Le Marec et Faury, 2013), mais aussi dans les billets des carnets, notamment lorsque l'énonciation à la première personne est adoptée, et quand le style d'écriture tend vers la forme orale.

10 Entrer dans le terrain par une analyse qualitative me permet de plonger dans les interfaces blogs en tant que « lieu habité » et non uniquement en tant que métadonnées privées de la présence numérique de l'auteur.e et de ses intentions d'un point de vue éditorial, scientifique et relationnel.

11 Enfin, j'identifie les carnets de thèse comme des lieux dans lesquels les doctorant.es parlent en leur nom et non pas au nom de leur laboratoire ou de leur direction. On écarte ainsi de l'étude des modèles d'utilisation des carnets comme sites de communication institutionnelle ${ }^{9}$ pour toucher à des questions liées à la présentation de soi et à la présence numérique des doctorant.e.s (Merzeau, 2010 ; Merzeau, 2009), ainsi qu'à l'effet des dispositifs sur les discours. Cette posture devient épistémologique (et politique), rendant possible le partage de l'expérience vécue et située de la recherche.

12 Ainsi, dans les carnets de thèse, tous les billets ne visent pas à " transmettre le savoir au grand public ». Le carnet accueille les écritures ordinaires de la recherche (Lefebvre, 2013), formelles ou informelles, normées ou non, et la discrimination entre les différentes formes de communication accueillies sur le blog n'est pas toujours explicite $^{10}$. Il en résulte que les savoirs partagés ne se déclarent pas toujours explicitement comme tels, de même que les témoignages de l'expérience de l'activité de recherche ne prétendent pas être des connaissances scientifiques, mais sont de précieux savoirs situés ${ }^{11}$, des savoirs d'expérience ou encore des savoirs de vie ${ }^{12}$. Autant de savoirs qui se construisent par l'écriture dans le carnet tout en étant en même temps partagés : en cela il s'agit de savoirs habituellement invisibles en vulgarisation appartenant à l'ordinaire ou l'infra-ordinaire de la recherche - et qui font dès lors, de fait, partie de la communication des doctorants. 


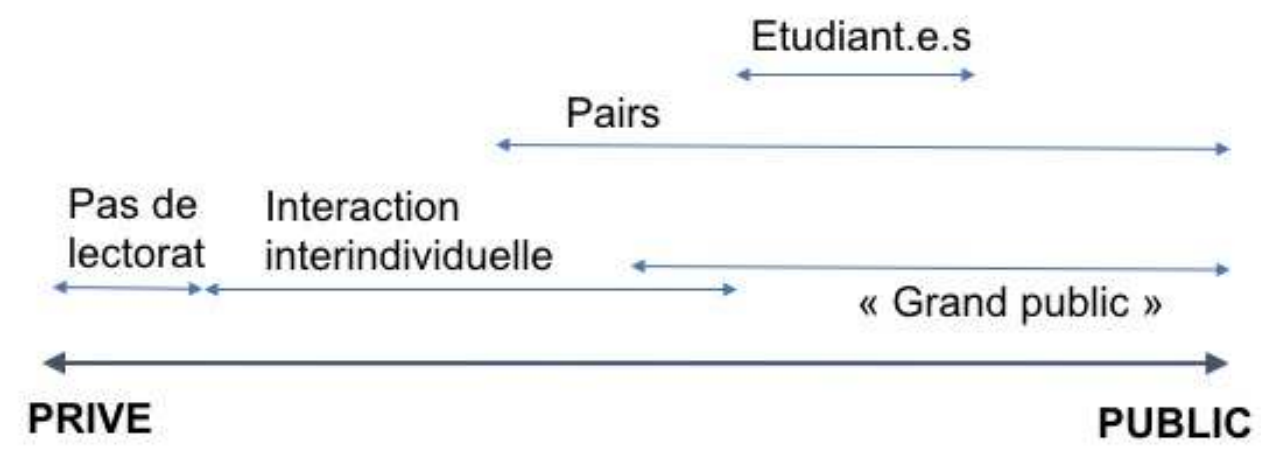

Figure 1 « Lectorats des carnets de recherche»

\section{Ce que les doctorant.es construisent, expriment et ce dont elles et ils témoignent dans les billets}

13 Les carnets de thèse sont donc des espaces intéressants que s'approprient les doctorant.es - et que parfois ils habitent -, en tant qu'espace potentiel, lieux de communication et mais aussi lieux d'inscription, d'expression, de partage de questionnements, de choix, de normes et de valeurs.

14 La typologie que je dessine vise à rendre compte de manière transversale de l'espace des possibles investi par l'ensemble des doctorant.es sur les carnets de thèse Hypothèses. Elle ne définit pas ce qu'est le carnet de thèse ni ce qu'il doit être. Je cherche plutôt à saisir des formations discursives (Foucault, 1969). Sans prétendre à l'exhaustivité, la liste de ces apparitions permet de rendre compte d'une certaine étendue, d'une sorte d'écosystème diversifié de types de billets ${ }^{13}$.

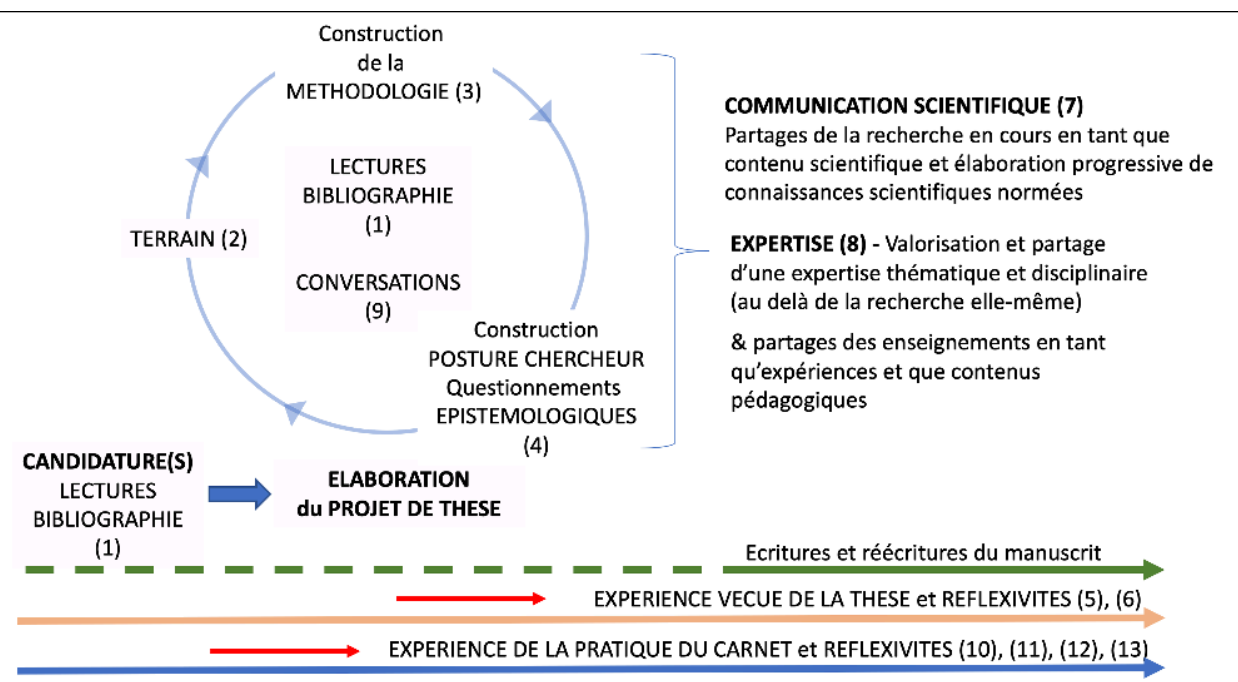




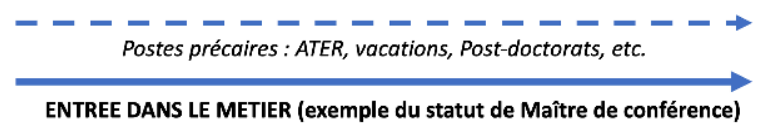

EXPERIENCE VECUE DE LA THESE (5), (6)

Figure 2 « Recherche doctorale et écritures de billets sur un carnet de thèse »

Légende de la figure 2 : Les numéros indiqués correspondent aux types de billets décrits en partie II: (1) Lectures ; (2) Terrain, (3) Méthodologie ; (4) Posture de chercheur et épistémologie ; (5) Expérience vécue ; (6) Emotions et corps ; (7) Communication scientifique ; (8) Expertise ; (9) Conversation ; (10) Soin du lectorat ; (11) Réflexivité écritures ; (12) Réflexivités sur le blogging scientifique ; (13)

Réflexivité rôle social du chercheur

Ce schéma représente les types de billets identifiés dans le référentiel du processus de recherche doctorale. En lien avec ces schématisations, je choisis d'explorer les billets rencontrés dans les carnets de thèse Hypothèses selon trois grandes catégories reliées à la mise en œuvre de la réflexivité, individuelle et collective, dans nos pratiques de recherche (Le Marec, 2002). La typologie est construite à partir de la question suivante : qu'est-ce qui est partagé par les doctorant.es dans leurs carnets?

\subsection{Le parcours de la recherche doctorale - emboîtements réflexifs}

\subsubsection{Projet de recherche individuel et expérience de la thèse}

16 Écrire sur sa propre recherche, parfois sous des formes proches de l'oral ou des formes que l'on pourrait dire "parlées", propres au blog, en adoptant l'énonciation à la première personne, peut être source de réflexivité par l'écriture ou par l'oralité (Jurdant, 2006; Gunthert, 2013; Maillot, 2018) : comprendre ce que l'on est en train de dire et de faire, et en retour, aller vers plus de justesse. On retrouve dans les carnets de thèse, sous la forme de billets, des témoignages de toutes les étapes représentées de manière cyclique dans le schéma 1 .

17 - Lectures $^{14}(1)$ : en amont de la recherche et tout au long de la construction de la thèse, les lectures et l'élaboration de bibliographie accompagnent les doctorant.es. Le partage de leurs perspectives sur les articles et ouvrages de leur champ contribue à la conversation scientifique et à leur positionnement dans le champ dans lequel elles et ils cherchent à entrer. (Figures 3 et 4 )

18 - Terrain ${ }^{15}$ (2) : dans certains billets, les doctorant.es documentent et racontent leur terrain de recherche. Il s'agit de témoignages de la construction de leur terrain de thèse, de la formulation d'un rapport au terrain ou encore de réflexions issues de l'expérience du terrain. (Figures 5 et 6 ) 
19 - Méthodologie ${ }^{16}$ (3) : les doctorant.es utilisent leur carnet de thèse pour partager des ressources et des questions sur la méthodologie de la recherche. Elles et ils explicitent leur démarche de recherche et partagent leurs retours d'expérience quant à la construction d'une thèse et à l'utilisation d'outils de la recherche (numérique, gestion de la bibliographie, etc.), leurs "trucs et astuces». Une forme de solidarité «entre doctorant.es » et plus largement avec les étudiant.es et « futurs entrant.es », s'exprime dans ces billets. (Figures 7, 8 et 9 )

20 - Posture de chercheur.e et épistémologie (4) : en lien avec les trois types de billets précédents, les doctorant.es partagent également des questionnements, notamment épistémologiques ${ }^{17}$, des doutes ${ }^{18}$, des incertitudes et des imprévus, et élaborent, en même temps qu'elles et ils la partagent, une posture de chercheur. ${ }^{19}$. (Figures 10,11 et 12)

21 - Expérience vécue de la thèse ${ }^{20}(5):$ les doctorant.es sont amenés à partager des récits de manière située dans leur expérience propre, liés à leur statut de doctorant.e et aux différentes étapes du parcours du doctorant.e, puis de la/du docteur.e. Elles et ils donnent à se représenter la thèse et la vie de doctorant.e. Ces billets s'inscrivent souvent dans l'ordinaire et l'infra-ordinaire de la recherche (Lefebvre, 2013 ; Perec, 1989) et peuvent être des lieux de développement d'une réflexivité individuelle. Dans ce type de billet, on trouve une narration de la thèse comme un sujet scientifique, qui se construit et avec lequel les doctorant.es entretiennent une relation : la thèse comme un parcours, ou en tant que moment de passage «à la suite " (voir Figure 2), du point de vue de l'entrée dans un métier. La thèse comme parcours se trace notamment par des billets portant sur les thèmes suivants : commencer la thèse, le quotidien de la thèse, points d'étapes et jalons, structuration de la thèse comme "sujet à présenter ", ce qui se passe "autour » de la thèse, la fin de la thèse, "l'après-thèse » et l'épreuve de la " condition de doctorant.e ». On note la place particulière de la soutenance en tant qu'expérience, qu'étape et rituel de "fin » de la thèse (qui marque parfois la fin du carnet de thèse). (Figures 13, 14, 15, 16 et 17)

- Billets portant sur les émotions et sur le $\operatorname{corps}^{21}(6)$ : quand les doctorant.es s'autorisent à parler d'émotions et du corps, la figure de chercheur.e qu'elles et ils construisent tranche avec la vision positiviste d'un chercheur à distance de son terrain qu'il cherche à objectiver : l'écriture témoigne qu'elles et ils sont touchés - voire transformés - par leur terrain (épistémologie du tact et du contact: Puig de la Bellacasa, 2012 ; Le Marec, 2013) et qu'elles et ils sont présent.es dans et à leur terrain (communication avec présence), en relation avec lui ${ }^{22}$ (épistémologie du lien). C'est l'expérience vécue qui fait émerger l'émotion et c'est le lieu du blog qui permet d'en recueillir le témoignage "autorisé ", « légitime ", du fait de l'habitude du récit de soi que le blog comme genre permet (Deseilligny, 2010). L'espace du blog, parce qu'il existe au sein de la pratique de recherche, et pas seulement comme lieu de communication des résultats de la recherche, s'inscrit dans de forts enjeux épistémologiques. J'avance que le carnet de thèse investi selon une fonction de témoignage de l'expérience vécue de la thèse transforme le rapport des doctorant.es à leur recherche. Cette culture du « récit de soi » propre au blog (en tant que genre), transposé à la recherche est propice à une dynamique réflexive proche de l'épistémologie du point de vue et des savoirs situés (Haraway, 1988 ; Harding, 1993 ; Faury, 2019). (Figure 18) 


\subsubsection{Inscrire sa recherche et s'inscrire soi-même dans une discipline, dans un champ}

Le carnet de thèse, en tant que site internet public, est un espace de communication au sens de la valorisation de l'accomplissement d'une activité professionnelle. Il permet aux doctorant.es d'être visibles et identifiés par leurs pairs, et de commencer à construire leur légitimité dans le champ (Bourdieu, 1976).

24 - Outil de communication et visibilité de leurs travaux ${ }^{23}(7)$ : les doctorant.es valorisent leur recherche, écrivent sur les communications qu'elles et ils font (en tant qu'expérience ou en en relayant le contenu dans un souci de centralisation de leurs informations), s'engagent dans une démarche de science ouverte (au sens de l'open access pour leur travail de recherche). (Figure 19)

- Traitement de l'actualité et partage de l'expertise ${ }^{24}$ (8) : par ces billets, les doctorant.es problématisent l'actuel, le quotidien et la culture populaire, à partir de leur perspective de recherche. Elles et ils donnent parfois un avis d'expert sur une question politique et investissent un rôle "d'intellectuel dans la cité " (Dacos et Mounier, 2010). En lien avec la posture de chercheur.e qu'elles et ils élaborent par le fait même d'écrire sur le carnet de thèse, les doctorant.es partagent du contenu et donnent à se représenter une recherche faite de questions vivantes ${ }^{25}$ plus que de certitudes. En tant qu'expert.es de leur discipline, elles et ils partagent aussi, le cas échéant, des contenus pédagogiques ou des retours réflexifs sur leurs enseignements ou sur les manières d'enseigner. (Figures 20, 21 et 22)

26 - Conversation scientifique ${ }^{26}$ (9) : la conversation scientifique induite par les carnets de recherche n'est pas toujours au cœur de la démarche, ni à la hauteur des attentes des doctorant.es ${ }^{27}$ - les billets des carnets de thèse recueillent en grande majorité peu ou pas de commentaires. Cependant on note aussi l'existence de quelques billets (et non pas seulement de commentaires) qui participent explicitement à la conversation entre pairs, prolongeant des discussions amorcées ailleurs (par courrier électronique, sur Twitter, In Real Life, ...). L'effet de socialisation des carnets de thèse ne passe pas toujours directement par l'espace numérique dédié au commentaire dans le carnet, mais par l'extérieur du carnet : il emprunte les voies des réseaux sociaux de chercheurs sur lesquels sont relayés leurs billets publiés au fil du temps (par eux-mêmes ou par leurs lecteurs). Twitter, Facebook, les recommandations par courrier électronique et les liens pointant d'un carnet de recherche à l'autre contribuent à ces liens sociaux et à la (re)connaissance des billets publiés. L'écriture sur les carnets de recherche est source d'une reconnaissance et d'une légitimité propre au réseau numérique que cette pratique construit, réseau structuré à partir des pratiques d'écritures et de lectures dans ces espaces numériques ${ }^{28}$. Ce réseau est composé notamment des pairs, collègues ou futurs collègues des doctorant.es carnetier.es, intéressés par les sujets traités. On note aussi l'émergence de la figure des doctorant.es carnetier.es qui se relient entre eux sur Twitter, en tant qu' «homologue» via leur pratique (partage d'une même expérience), plus que par leur sujet ou leur discipline d'appartenance (nouvelles affinités). (Figure 23) 


\subsection{Ouverture de la recherche}

27 Les carnets de recherche sont des espaces potentiels de partages de contenus scientifiques construits ou en cours d'élaboration. Ils participent à l'ouverture de la recherche auprès d'un lectorat divers (Figure 1), constitué de spécialistes, de communautés d'intérêt ou du "grand public» selon les billets et le sujet qu'ils abordent, leur accessibilité, leur adressage éventuel et l'adaptation, ou non, de la forme au fond et au public. Le lectorat se construit à partir des ressorts de la sociabilité du web, de l'hospitalité des carnets de thèse en tant qu'espaces numériques habités par les doctorant.e.s et la constitution de liaisons numériques à partir des contenus proposés dans les carnets et de leur circulation en ligne, notamment via les réseaux sociaux (Casilli, 2010): les billets écrits sans public a priori trouvent ainsi leurs lecteurs, notamment par les affinités électives qui se tissent via les moteurs de recherche et l'utilisation par les doctorant.e.s de mots-clés et de métadonnées pour qualifier leurs billets.

28 Ainsi, les témoignages de doctorant.es carnetier.es (dans les entretiens menés et lors de la journée des 10 ans de la plateforme Hypothèses) montrent qu'elles et ils écrivent sans être d'abord ${ }^{29}$ certains d'être lus, ni sans savoir précisément à qui elles et ils s'adressent. Puis elles et ils trouvent des signes de cette lecture effective (statistiques $\mathrm{du}$ blog, relais sur les réseaux sociaux, retour de lecteurs in real life). L'adressage se précise parfois au fil du temps mais ne garantit pas la réception et chaque billet peut s'adresser à un public imaginé différent. Ainsi Karim Hammou, sociologue du rap, fait-il par exemple la typologie suivante de ses lecteurs destinataires de ses billets pendant 9 ans de blogging sur son carnet personnel: « amateurs de rap ", " enquêtés ", " grand public », « hip-hop studies », « carnetiers », « académie » (Hammou, 2019).

\subsubsection{Soin du lectorat}

29 Le blog de recherche est un espace mixte en termes de "publics" qualifiés de «lectorats» avec lesquels la conversation est le plus souvent silencieuse, mais qui peuvent être des lecteurs fidèles du carnet (Dacos et Mounier, 2010), tels que les statistiques disponibles pour chaque carnet peuvent le mettre en évidence ${ }^{30}$.

Selon les carnets de thèse, la prise en compte du lectorat ${ }^{31}$ et la conversation (même silencieuse - Dacos et Mounier, 2010) sont plus ou moins perceptibles : il peut s'agir de marquer explicitement des étapes et de faire des efforts de lisibilité et d'explicitation quant aux intentions des billets et du carnet (ne pas «écrire pour soi seul»), ce peut être s'adresser explicitement au lectorat dès le titre ou l'accroche, ou encore engager une conversation et le questionner. Dans les billets, on retrouve la trace de la prise en compte du lectorat et de la relation que les carnetier.es souhaitent établir ou entretiennent; en engageant une conversation ou en remerciant les lecteurs de leur présence par exemple. (Figures 24 et 25)

\subsubsection{Effet réflexif de l'ouverture et de l'intégration d'un « autre »}

31 Je considère a priori que les carnets de thèse sont les espaces potentiels d'une recherche située en mouvement, en interrogations, sur le terrain, vivante, en évolution, incarnée. Ils permettent de saisir la présence du rapport des doctorant.e.s à leur sujet, à leur terrain, à leurs objets, rencontres, à leurs lectures. Ils contribuent à 
mon sens à l'émergence de différentes formes de réflexivités : sur l'écriture exposée l'intégration de la possibilité de l'autre dans le propos (11) et sur l'activité de blogging elle-même (12).

Le geste d'écrire, les objectifs, normes et situations de communication qui y sont associées font parfois l'objet de billets réflexifs (11). On trouve par exemple des réflexions sur les écritures de la thèse, la co-écriture, sur les écrits destinés aux pairs et notamment sur les articles ou la thèse elle-même, ou encore sur l'écriture pour d'autres publics et notamment pour le " grand public ». (Figure 26)

L'activité de carnetier.e elle-même est source de questions et de réflexivités dans les carnets de thèse (12) : pourquoi blogguer ? À partir de bilans d'étape de la pratique du carnet, articulés parfois à des étapes du parcours de recherche, les doctorant.es s'interrogent sur le sens de la pratique du blog en articulation avec les enjeux et avec le sens de leur pratique de recherche. La réflexivité sur l'expérience de blogging s'appuie sur l'explicitation de la place de cette pratique dans leur activité de recherche et donc aussi des choix, des attentes, des normes et des valeurs qui amènent les doctorant.es à consacrer du temps à l'écriture dans leur carnet de thèse. Cette réflexivité peut être limitée par un enjeu de légitimation de la pratique du blogging qui mobilise les normes et les valeurs de la recherche pour justifier son existence, et non pas (seulement) le sens de la pratique de carnetier.e pour les doctorant.es. (Figure 27)

Ainsi, l'écriture ouverte vers d'autres publics que les pairs ou que des chercheur.es d'autres disciplines amène les doctorant.es (souvent après un certain temps passé à écrire sur leur carnet) à s'interroger sur les enjeux et les effets de l'écriture ouverte ou "grand public». Il s'agit soit d'un retour d'expérience (acquise par la pratique du carnet) sur les changements constatés dans leur écriture de billets et même d'articles scientifiques, soit d'interrogations explicites sur ce qui distingue le fait de s'adresser aux pairs ou à des non-spécialistes. On touche, de manière plus ou moins explicite dans ces billets réflexifs, à un questionnement sur la place et le rôle des chercheur.ses dans les relations science-société.

\section{A la croisée des marges : une écriture plus libre et réflexive au bénéfice de la recherche}

La pratique du carnet de thèse est une pratique de communication au fil de l'activité de recherche doctorale, qui agit sur la manière dont les jeunes chercheur.es construisent leur activité de recherche, sur leur visibilité, et le cas échéant, sur leur notoriété rattachée à un champ de recherche (notoriété scientifique dans le champ - si et seulement si la qualité scientifique suit - et notoriété publique hors du champ), sur l'inscription des doctorant.es dans un réseau professionnel, non réduit aux interactions numériques, et sur la construction d'un public pour leur recherche.

Par la pratique du carnet de thèse, les doctorant.es partagent à la fois ce qu'ils et elles savent et cherchent, s'éloignant ainsi d'une vulgarisation classique partant de « ce que l'on sait déjà » (Jurdant, 1969) ou qui tisserait un lien entre sachant et " ignorants ». Les doctorant.es ne "diffusent" pas la "vérité scientifique " mais partagent y compris leurs incertitudes, leurs questions, leurs errances... L'écriture sur un blog et la logique des réseaux sociaux nous font considérer également une "vulgarisation " qui ne se déploie pas selon un modèle diffusionniste de communication (émetteur-canal- 
récepteur) vers un public « en manque de science » (Bensaude-Vincent, 2010), mais par la rencontre avec un public, ou lectorat, qui se constitue à partir du discours partagé, et plus particulièrement des métadonnées thématiques qui lui sont associées (Casilli, 2010). Le lectorat ne préexiste pas, les doctorant.es ne viennent pas délibérément combler un « déficit » de discours sur leur sujet de recherche, mais s'investissent selon une multitude de motivations pour la vulgarisation (Maillot, 2018), de logiques d'écritures dans les carnets de recherche (Poupardin et Faury, 2019 à paraître), d'enjeux, notamment idéologiques (Jurdant, 1969 et 2008). Le sens que prend pour elles et eux l'investissement dans les carnets de recherche peut entrer en conflit de normes et de valeurs - au sein de relation de pouvoir - avec la légitimité que l'institution et les collègues (notamment les directeurs de thèse) accordent à cette pratique.

Les carnets de thèse proposent tout à la fois de nouvelles figures du vulgarisateur ou de la vulgarisatrice et de nouvelles situations de vulgarisation, à partir d'une implication directe de locuteurs immergés dans la pratique quotidienne de la recherche : brouillant les frontières de ce que l'on peut dire ou ne pas dire, montrer ou ne pas montrer de la science-en-train-de-se-faire (Blanchard, 2008 et 2009). Les doctorant.es - mobilisé.es par ailleurs dans d'autres dispositifs de vulgarisation (Faury et Maillot, 2019) - ne se positionnent pas seulement en tant qu'experts, ni ne parlent uniquement de leur sujet de thèse : elles et ils partagent leur expérience, au fil du temps, selon une ligne éditoriale pas toujours prédéfinie, qui se construit au fil de leurs envies, de leurs besoins, du temps disponible. Elles et ils sont présents à une forme de communication qui ne leur est pas commandée, ni prescrite : elles et ils habitent, à leur manière et parfois avec leur propre voix, leur propre lieu qui s'extrait - au moins en partie - des espaces de communication institutionnelle dans lesquels elles et ils sont pris ou peuvent choisir d'intervenir, selon des règles et des normes qu'elles et ils endossent et incarnent (Faury, 2012). Elles et ils prennent la parole dans leur carnet de thèse avec différents styles ${ }^{32}$ selon ce qu'elles et ils projettent de ce qu'est un doctorant.e, de ce qu'elle et il peut dire ou non publiquement, vraisemblablement aussi selon le contexte dans lequel elles et ils travaillent (laboratoire, directeur de thèse, etc.) : selon leur espace mental de la recherche (Faury, 2012) mais aussi selon leur représentation de la pratique du carnet de recherche, elle-même influencée par les carnets qu'elles et ils peuvent lire et selon ce qu'elles et ils en perçoivent dans les discours portés par la plateforme Hypothèses.

Dans le cas des carnets de thèse, les doctorant.es se situent dans un espace " entre ", à la croisée de plusieurs dispositifs : entre l'espace personnel et l'espace normé de la recherche; entre l'espace personnel et l'espace normé du blog Hypothèses - qui s'institutionnalise de fait de plus en plus en accord avec les normes de l'activité de recherche; entre l'espace du blog et l'espace de la recherche comme pratique professionnelle institutionnalisée. Cette situation intermédiaire marginale à plusieurs titres (marge de recherche, marge de la thèse, marge des blogs) laisse apparaître une certaine liberté de ton et la possibilité d'une écriture "autre», qui contraste en particulier avec les écrits formels des articles scientifiques revus par les pairs, et qui est investi sur un temps le plus souvent "en plus » de tout ce qui est attendu des doctotant.es au cours de leur thèse (articles, manuscrit, enseignements, etc.). L'analyse des carnets de thèse montre de surcroît que cette vision d'un "à côté » (" en plus » ou " au détriment») du temps du carnet de recherche ne tient pas tout à fait dans la mesure où l'écriture du carnet nourrit la réflexivité du travail de recherche. Cette liberté située, et par rapport aux écritures centrales de la recherche, est en fait elle- 
même inspirée et contrainte par les possibilités techniques et par les normes de l'écriture du blog (Couleau et Hellégouarc'h, 2010) - c'est-à-dire par le dispositif du « carnet».

C'est justement le croisement entre deux dispositifs - la thèse et le carnet de thèse - qui me parait particulièrement intéressant du fait des porosités que leur cohabitation crée entre les différentes écritures des doctorant.es : billets, articles, manuscrits, etc. Ces porosités ont des conséquences épistémologiques (Faury, 2019). Les carnets de thèse mis en regard d'autres formes de publications (articles, manuscrit de thèse, etc.) mettent en évidence de manière tracée et explicite le continuum décrit par D. Jacobi (1984) au sujet de la vulgarisation et inscrivent les écrits intermédiaires de la recherche, habituellement non partagés en ligne, dans un espace ouvert, dans l'esprit de «l'open science ».

L'analyse des billets de carnets de recherche réactualise enfin la thèse de $B$. Jurdant selon laquelle la vulgarisation porterait avant tout un potentiel de réflexivité au bénéfice des chercheur.es, en l'occurrence des doctorant.es eux-mêmes. Par la vulgarisation ou par l'écriture dans un carnet de thèse ouvert, espace numérique où elles et ils parlent (Faury, 2019; Jurdant, 2006; Goody, 1986), les doctorant.e.s font l'expérience de la réflexivité. Ce mouvement réflexif part de la pratique de thèse et $\mathrm{y}$ revient.

41 Ainsi, le déploiement de discours dans l'espace du carnet et l'expression écrite incluant un effet de distance - de cette réflexivité peut avoir des conséquences majeures liées à la recherche présentée :

1/ en renforçant la scientificité de la recherche en cours des doctorant.es, par le mouvement réflexif qui peut être déployé dans l'espace du carnet, situant la recherche, le rapport des doctorant.es à leur terrain et la manière dont elles et ils prennent la parole en tant que scientifiques ;

2/ pour la recherche en STS (Science and Technological Studies), les carnets de thèse offrent de nouveaux types de savoirs sur la pratique de la recherche et sur les pratiques de communication des chercheurs. La réflexivité ordinaire (Jeanneret, 2010) que développent et partagent les doctorant.e.s, est une source précieuse de savoirs " méta » pour la recherche en sciences humaines et sociales, et notamment pour la recherche en études de sciences par l'entrée des sciences de l'information et de la communication.

BIBLIOGRAPHY

\section{Articles et ouvrages}

Bourdieu, Pierre, «Le champ scientifique », Actes de la recherche en sciences sociales, 1976 (2), n²-3, p. 88-104. 
Bensaude-Vincent, Bernadette, «Splendeur et décadence de la vulgarisation scientifique ", Questions de communication, 2010, n 17, p. 19-32. En ligne : http:// questionsdecommunication.revues.org/368

Casilli, Antonio A., Les liaisons numériques - Vers une nouvelle sociabilité, Paris, Seuil, 2010.

Couleau, Christelle et Pascale Hellégouarc'h, « Les blogs - Écritures d'un nouveau genre ?», Itinéraires. Littérature, textes, cultures, 2010, $\mathrm{n}^{\circ} 2$.

Faury, Mélodie, « Carnets de thèse et écriture de soi dans la recherche », Revue de la BnF, 2019, n58, p.73-81.

Dacos, Marin, et Pierre Mounier, « Les carnets de recherche en ligne, espace d'une conversation scientifique décentrée ", dans Jacob Christian (dir.), Lieux de savoir, Gestes et supports du travail savant, Paris, Albin Michel, 2010. En ligne : http://archivesic.ccsd.cnrs.fr/sic_00439849/document Deseilligny, Oriane, « Le blog intime au croisement des genres de l'écriture de soi ». Itinéraires. Littérature, textes, cultures, 2010, $\mathrm{n}^{\circ} 2, \mathrm{p}$ 73-82.

Faury, Mélodie et Lionel Maillot, « Repenser les formations doctorales - De la communication efficace à la communication incarnée ", La lettre de l'Ocim, 2019, nº181, à paraître.

Faury, Mélodie, « Parcours de chercheurs. De la pratique de recherche à un discours sur la science : quel rapport identitaire et culturel aux sciences? ", Thèse, École normale supérieure de Lyon, 2012. En ligne : https://tel.archives-ouvertes.fr/tel-00744210/document (consulté le 5 octobre 2018).

Foucault, Michel, L'archéologie du savoir, Paris, Gallimard, 1969.

Goody, Jack, La logique de l'écriture : aux origines des sociétés humaines, Paris, Armand Colin,1986.

Harding, Sandra, « Rethinking Standpoint Epistemology: What is Strong Objectivity? », dans Linda Alcoff et Elizabeth Potter (dir.), Feminist Epistemologies, New York \& London, Routledge, 1993. pp. 49-82.

Haraway, Donna, « Situated knowledges: the science question in feminism and the privilege of partial perspective ", Feminist Studies, 14 (3), 1988, pp. 575-599.

Jacobi, Daniel, « Recherches sociolinguistiques et interdiscursives sur la diffusion et la vulgarisation des connaissances scientifiques ». Thèse d'État, Besançon, Université de Franche Comté, 1984.

Jeanneret, Yves, «Le statut des savoirs ordinaires dans l'analyse des pratiques de communication ", Methis. Interdisciplinarité en sciences humaines et sociales, 2010, n³, pp 21-50. En ligne : https:// popups.uliege.be:443/2030-1456/index.php?id=362.

Jurdant, Baudouin, « Entre science et société : les ambiguïtés de la vulgarisation », dans JeanPierre Ali, Bernard Ancori et Pierre Petit (dir.), Sciences en société au XXI ${ }^{e}$ siècle-autres relations, autres pratiques, Paris, CNRS Editions, 2008.

Jurdant, Baudouin, « Parler la science ? », Alliage, 59, 2006, p. 57-63. En ligne : http:// www.tribunes.com/tribune/alliage/59/page6/page6.html

Jurdant, Baudouin, « Le désir de scientificité », Alliage, 41-42, 1999, p. 147-155. En ligne : http:// revel.unice.fr/alliage/index.html?id=3932

Baudouin Jurdant, « Vulgarisation scientifique et idéologie », Communications, 14, 1969, p. 150-161. 
Latour, Bruno et Steve Woolgar, La vie de Laboratoire. La production des faits scientifiques, Paris, La Découverte, 1996.

Macé, Marielle, Styles. Une critique de nos formes de vie, Paris, Gallimard, 2016.

Le Marec, Joëlle, « Le public, le tact et les savoirs de contact », Communication \& langages, 2013 (1), $n^{\circ} 175$, p. 3-25. En ligne : https://www.cairn.info/revue-communication-et-langages1-2013-1page-3.htm

Le Marec, Joëlle, Faury, Mélodie, « Communication et réflexivité dans l'enquête par des chercheurs sur des chercheurs ", dans Jacques Béziat (dir.), Analyse de pratiques et réflexivité: Regards sur la formation, la recherche et l'intervention socio-éducative, L'Harmattan, 2013, p. 153-166.

Le Marec, Joëlle, « Situations de communication dans la pratique de recherche : du terrain aux composites ", Études de communication, 25, 2002. En ligne : $\underline{\text { http://journals.openedition.org/edc/ }}$ 831.

Lefebvre, Muriel, « L'infra-ordinaire de la recherche. Écritures scientifiques personnelles, archives et mémoire de la recherche ", Sciences de la société, 89, 2013, p. 3-17. En ligne : https:// journals.openedition.org/sds/203

Macé, Marielle, Styles. Une critique de nos formes de vie, Paris, Gallimard, 2016.

Maillot, Lionel, « La vulgarisation scientifique et les doctorants : Mesure de l'engagement exploration d'effets sur le chercheur », Thèse en Sciences de l'information et de la communication, Université de Bourgogne - Franche Comté, 2018.

Merzeau, Louise, « La présence plutôt que l'identité ». Documentaliste-Sciences de l'Information, 47, 1, 2010, p. 32-33.

Merzeau, Louise, « Présence numérique : les médiations de l'identité », Les Enjeux de l'information et de la communication, 1, 2009, p. 79-91. En ligne : https://www.cairn.info/revue-les-enjeux-de-linformation-et-de-la-communication-2009-1-page-79.htm

Paveau, Marie-Anne, «Le désir épistémologique », Semen - Revue de sémio-linguistique des textes et discours, 29, 2010, p. 7-13.

Elsa Poupardin et Mélodie Faury, «Écrire dans un carnet Hypotheses.org : l'inscription d'une pratique de communication dans l'activité de recherche », 2019, à paraitre.

Piron, Florence, « Méditation haïtienne. Répondre à la violence séparatrice de l'épistémologie positiviste par l'épistémologie du lien », Sociologie et sociétés, Vol. XLIX, n 1 1, 2017, pp. 33-60.

Puig de la Bellacasa, Maria, «Technologies touchantes, visions touchantes. La récupération de l'expérience sensorielle et la politique de la pensée spéculative. » dans Penser avec Donna Haraway, Paris, Presses Universitaires de France, 2012.

Puig de la Bellacasa, Maria, Politiques féministes et construction des savoirs. "Penser nous devons"!, Paris, L'Harmattan, 2013.

Perec, George, L'infra-ordinaire, Paris, Seuil, 1989.

Schwartz, Olivier, «L'empirisme irréductible. La fin de l'empirisme? », dans Anderson Nels (dir.), Le hobo, sociologie du sans-abri, Paris, Armand Colin, 2011 (1923), p. 335-384.

Thiault, Florence, « Recherche indigène et familiarité avec l'objet de recherche ", Études de communication, 32, 2009, p. 2-9. 


\title{
Billets
}

Blanchard, Antoine, « Comment montrer la "science en train de se faire” ?", 31 mai 2008, Disponible à l'adresse : http://www.enroweb.com/blogsciences/index.php?post/ 2008/05/31/261-comment-montrer-la-science-en-train-de-se-faire.

Blanchard, Antoine, « Les blogs de science, un nouvel acteur des relations science-société », 27e Congrès de l'AMCSTI. Sciences, innovation et société : quelles réponses apporter ?, 2009, Cherbourg, France : Association des musées et centres de culture scientifique, technique et industrielle, $\mathrm{p}$. 46-47. Disponible à l'adresse $:$ https://hal.archives-ouvertes.fr/hal-01265183.

Faury, Mélodie et Giraud, Frédérique, « Le carnet de thèse ", Espaces réflexifs (carnet de recherche), 13 février 2012. En ligne : http://reflexivites.hypotheses.org/641

Gunthert, André, « Le blogging académique, entre art et science ", L'atelier des icônes (Blog), 14 octobre 2013. En ligne : http://histoirevisuelle.fr/cv/icones/2820

Hammou, Karim, « Le carnet de recherche comme « lieu propre » numérique », Sur un son rap (carnet de recherche), 11 janvier 2019. En ligne : https://surunsonrap.hypotheses.org/3896 Jurdant, Baudouin, « Communication scientifique et réflexivité », Espaces réflexifs [carnet de recherche], 22 février 2012. En ligne : http://reflexivites.hypotheses.org/695

\section{APPENDIXES}

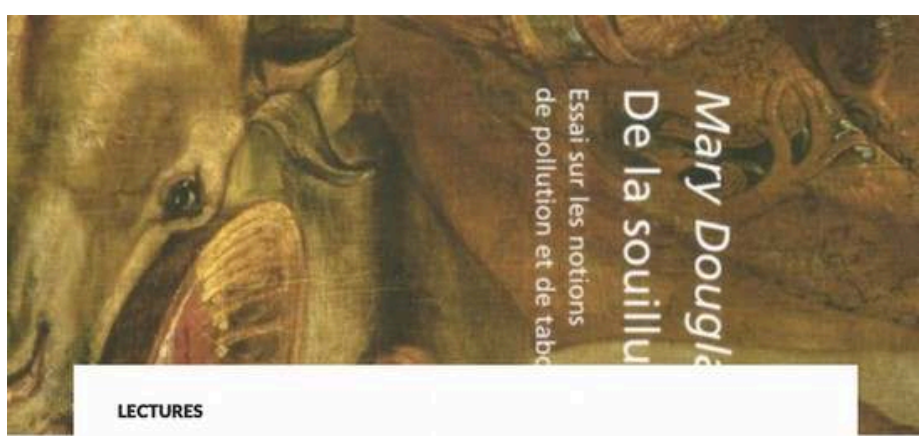

\section{UNE LECTURE DE...DE LA SOUILLURE, MARY DOUGLAS \\ Q 05/12/2017 i ANAIISMARTIN LAISSERUN COMMENTAIRE}

\begin{abstract}
Les mois passent et l'architecture du carnet se construit. C'est au tour de ce billet d'inaugurer une nouvelle catégorie : Lectures (même si en fait je triche un peu car un billet y est déja répertorié). Elle sera l'espace dans lequel je proposerai une lecture d'ouvrages et/ou d'articles en faisant notamment un point sur cequ'ils m'ont apporté.
\end{abstract}

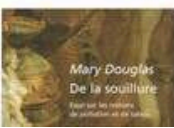

C'est assez rapidement et sans trop réfléchir que j'ai décidé d'inaugurer cette catégorie par un livre qui m'a à la fois beaucoup interrogée et

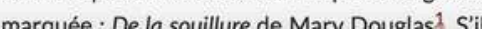

Figure 3 « Partager une lecture d'ouvrage »

Source : Anaïs Martin, « Une lecture de... De la souillure, Mary Douglas », Entre le zist et le zest (carnet de thèse). https://zistetzest.hypotheses.org/346. 


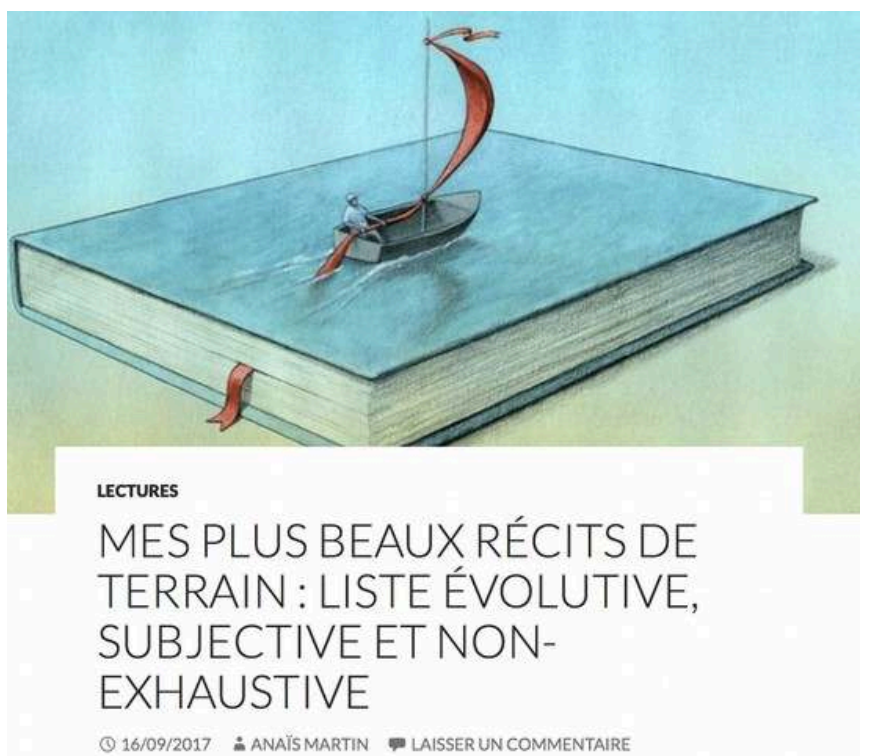

Parce qu'on découvre souvent l'anthropologie par les récits de terrain de chercheur.e.s plus ou moins contemporain.e.s de nous, et parce qu'en tant qu'anthropologue, on garde par la suite cet attrait pour les ethnographies (du moins c'est mon cas), j'avais envie de créer ce billet qui sera amené a évoluer au gré de mes lectures et de mes envies. II contiendra à la fois des récits ethnographiques au sens classique du terme, des ouvrages issus de

Figure 4 « Partager une bibliographie »

Source : Anaïs Martin, « Mes plus beaux récits de terrain : liste évolutive, subjective, et non exhaustive ", Entre le zist et le zest (carnet de thèse). https://

zistetzest.hypotheses.org/321.

\title{
ENCHANT(I)ER
}

\author{
[ENCHANT(I)ER] LA MAISON \\ DE L'HISTOIRE DE L'EAU (2) \\ (1) 01.09.2014 ¿ STÉPHANIEMESSAL LAISSER UN COMMENTAIRE
}

La maison de l'histoire de l'eau a été ainsi nommée car en son sein, on trouve un lavoir que le petit groupe à la tâche (dans le cadre de la réhabilitation de la Villa Megglé) espère voir un jour remplir sa fonction en se remplissant d'eau de nouveau. En attendant, quelques étudiants s'occupent de " murer " la façade de ce lavoir. Pas question de boucher la façade tel que pourrait le laisser entendre l'expression " murer " ! Il est plutôt question de jouer avec les matériaux disponibles, la lumière et les effets d'ouverture et de fermeture pour mettre en valeur l'ensemble : bref, le lavoir se fait ravaler la façade ! Le résultat est aussi surprenant que ludique.

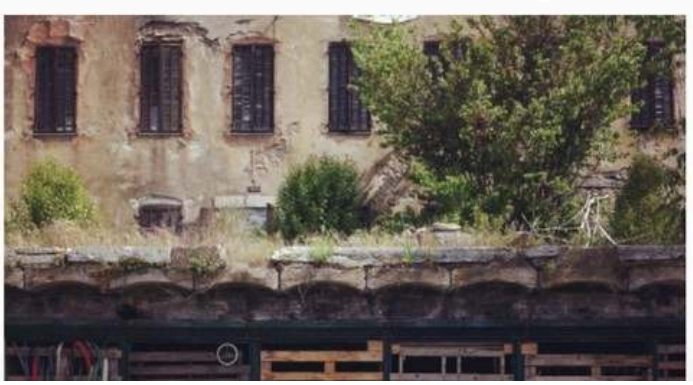


Figure 5 « Documenter et raconter le terrain »

Source : Stéphanie Messal, « [Enchant(i)er] La maison de l'histoire de l'eau (2)».

Misanthropologue (carnet de thèse). https://misanthropologue.hypotheses.org/1478.

\section{Le corps vivant du corpus}

Quoi qu'on en dise, la recherche en sciences humaines butte sur une limite très précise : la nécessité de faire parler les gens pour produire un corpus (entretiens, questionnaires). Cette demande est vite épuisante pour le chercheur, épuisée quant à ses possibilités d'extension, et corrompue dans son fonctionnement même qui la rend socialement artificielle, étant précisé que les sciences humaines ne peuvent généralement bénéficier de l'effet laboratoire qui prévaut souvent dans les sciences de la nature. Cependant, la société actuelle étant toujours plus, quoi qu'on en pense, une société du commentaire, de la conversation et de la réaction, de l'écriture (textos, messages, forums, pages web) et de la conservation (multiplication des traces par capture d'images, enregistrement vidéo, historique, archivage systématique, etc.), il ne sera bientôt plus nécessaire pour le chercheur, partout où ce l'était encore, de provoquer soi-même son corpus ni même de l'enregistrer, mais uniquement de le choisir et de le prélever. Ce qui ne relève pas moins d'un art délicat. Les sciences humaines pourront déployer alors leur vraie nature : d'être des sciences du corpus, foncièrement discursive (y compris quand elles ne s'appuient que sur un corpus d'images) et historique à la fois. Je m'aperçois qu'il y a là une constante de mes recherches : le désir d'établir un corpus qui soit aussi scientifiquement nécessaire que naturel, dans des

Figure 6 « Construire un rapport au terrain »

Source : Sébastien Marlair , « Le corps vivant du corpus », Postures \& récits (carnet de thèse). https://postures.hypotheses.org/302. 


\title{
Genèse d'un projet de recherche (Master)
}

\begin{abstract}
PAR XAVIER REGNIER - 13/06/2018
Cet article est à la fois un brouillon très personnel du projet de recherche que je dois bientôt rendre et un témoignage que j'espère utile pour ceux qui songent à s'engager en Master recherche. Sans prétention, l'expérience du mémoire de Master 1 et la lente maturation de mon projet actuel, ainsi que la lecture attentive et appliquée de l'article d'Émilien Ruiz, m'ont amené à poser ma réflexion, et à vouloir la partager. Il s'agit donc plus du cheminement que du projet final.
\end{abstract}

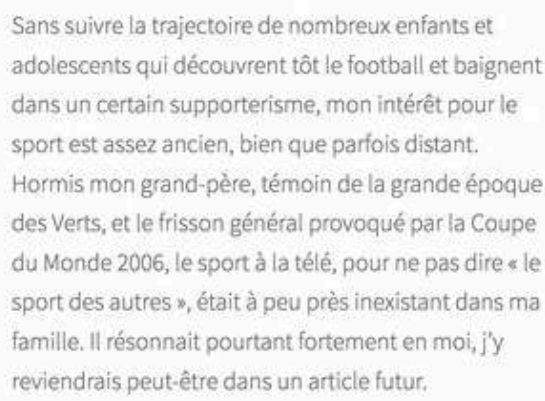

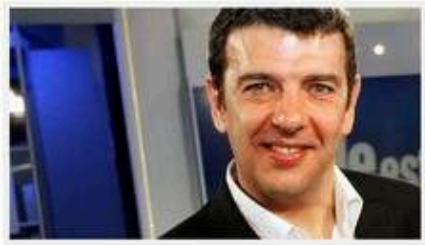

Thierry Grardi, auteur de mespremiers frissons sportitis

Source: Sport24-Lefigoro

Pour autant, je ne me suis penché sur l'objet historique « sport » que depuis les environs du mois de

Figure 7 «Partager la méthodologie pour les futurs chercheurs »

Source : Xavier Regnier, « Genèse d'un projet de recherche (Master)», Au miroir du sport (carnet de thèse). https://miroirsport.hypotheses.org/102.
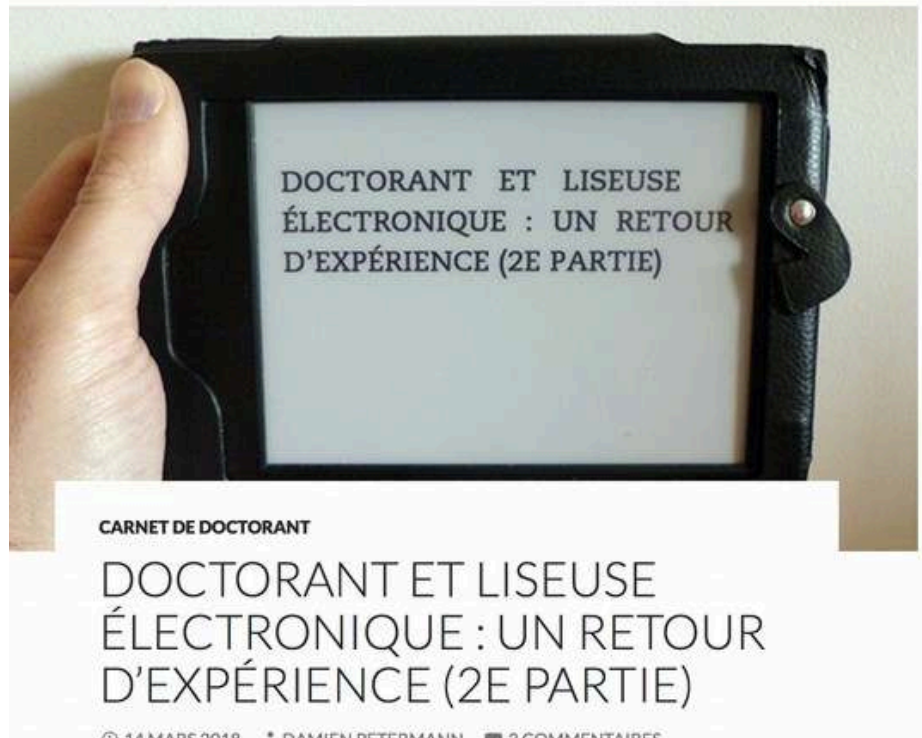

(1) 14 MARS $2018 \div$ DAMIEN PETERMANN $\approx 2$ COMMENTAIRES

Ce billet est la deuxième partie du diptyque consacré à mes usages de la liseuse électronique. Vous pouvez lire la première partie ici.

Passons maintenant aux usages qui concernent la relecture :

Figure 8 « Partager un retour d'expérience»

Source : Damien Petermann, « Doctorant et liseuse électronique : un retour 
d'expérience ( $2^{\mathrm{e}}$ partie) », L'image de Lyon (carnet de thèse). https:// imagelyon.hypotheses.org/820.

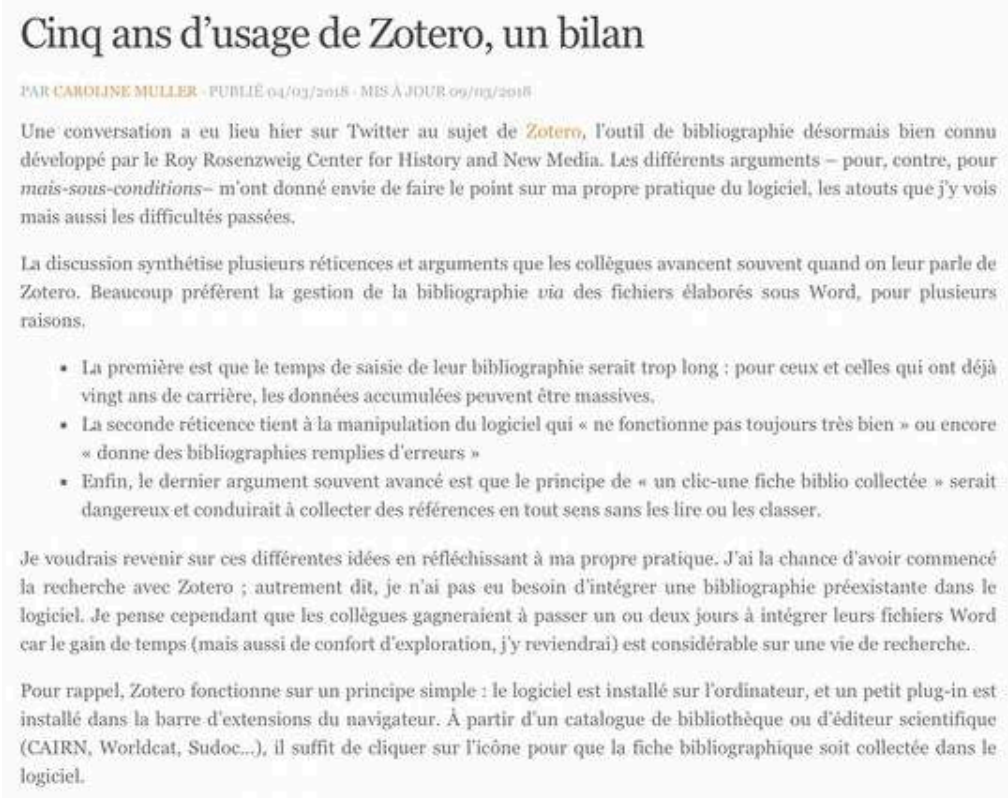

- La première est que le temps de saisie de leur bibliographie serait trop long : pour ceux et celles qui ont déja vingt ans de carrière, les données accumulées peuvent être massives.

- La seconde réticence tient à la manipulation du logiciel qui $\approx$ ne fonctionne pas toujours très bien * ou encore * donne des biblicgraphies remplies d'erreurs :

- Enfin, le dernier angument souvent avancé est que le principe de * un clic-une fiche biblio collectée * serait dangereux et conduirait à collecter des références en tout sens sans les lire ou les classer.

Je voudrais revenir sur ces différentes idées en réfléchissant à ma propre pratique. J'ai la chance d'avoir commencé la recherche avec Zotero ; autrement dit, je n'ai pas eu besoin d'intégrer une bibliographie préexistante dans le logiciel. Je pense cependant que les collègues gagneraient à passer un ou deux jours à intégrer leurs fichiers Word car le gain de temps (mais aussi de confort d'exploration, jy reviendrai) est considérable sur une vie de recherche.

Pour rappel, Zotero fonctionne sur un principe simple 1 le logiciel est installé sur lordinateur, et un petit plug-in est installé dans la barre d'extensions du navizateur. À partir d'un catalogue de bibliothèque ou d'éditeur scientifique (CAIRN, Worldcat, Sudoc...). il suffit de cliquer sur licône pour que la fiche bibliographique soit collectée dans le logiciel.

\section{in}

Figure 9 «Partager l'expérience des outils numériques : ici Zotero » Source : Caroline Muller, « Cinq ans d'usage de Zotero, un bilan », Acquis de conscience (carnet de thèse). https://consciences.hypotheses.org/1184.

\section{Position du chercheur et ethnographie multi-située}

L'article désormais classique de Georges E. Marcus, "Ethnography in/Of the World System: The Emergence of Multi-sited Ethnography ${ }^{1}$ propose un riche cadre de référence pour penser mon positionnement et le type d'ethnographie que je tiens à développer.

La réflexion de type méthodologique développée par Marcus dans cet article fait le point sur un champ ethnographique qui ne veut/peut plus dissocier " lifeword and system $^{2}$. Il rappelle très justement que la plupart des logiques culturelles reprises par l'anthropologie sont en partie constituées " within sites of the so-called system (i.e. modern interlocking institutions of media, markets, states, industries, universities - the worlds of elites, experts, and middle classes) ${ }^{3}$. Mais si le lien entre " lifeworlds of subjects and the system " ne tient plus, quel peut être la visée de l'ethnographie qui reste une discipline attachée à développer un point de vue local et proche des sujets étudiés (la fameuse injonction, " suivre les gens »). Pour Georges Marcus, il s'agit de 
Figure 10 « Construire et partager une posture de chercheur »

Source : Sandrine Teixido, "Position du chercheur et ethnographie multi-située ", Agir en musique (carnet de thèse). https://musicagir.hypotheses.org/46.

\title{
La ruée vers la donnée
}

Epistémologle de la donnée web en sclences sociales

\section{Quelques enjeux épistémologiques de l'analyse du web et des big data}

\author{
Je romps enfin le silence de ce carnet dont je n'ai guère eu le temps de m'occuper \\ ces derniers temps. J'ai beaucoup écrit pour la thèse mais rien de tout cela n'est \\ Eglantine \\ 9 jullet 2015 \\ Billets \\ visible pour le moment. Voici donc une « petite » synthèse de l'un des sujets qui \\ m'a occupée...

\begin{abstract}
La cinquième édition de la conférence « Document numérique et société » avait cette année pour thème « Open data, Big data : quelles valeurs, quels enjeux ? " Comme les enjeux épistémologiques des big data faisaient explicitement partie des axes de la conférence, j'ai décidé d'y présenter où j'en était de ma réflexion sur ce point.

L'enjeu n'était donc pas de proposer un éclairage sur les enjeux politiques, éthiques et sociaux des big data, qui sont déjà largement discutés par
\end{abstract}

Figure 11 «Construire et partager un questionnement épistémologique » Source : Eglantine Schmitt, « Quelques enjeux épistémologiques de l'analyse du web et des big data ", La ruée vers la donnée (carnet de thèse). https://bigdata.hypotheses.org/ 81. 


\section{De la sérendipité, en particulier.}

La " sérendipité " est à la mode depuis quelques années en sciences humaines. En suivant la directrice de recherches du CNRS, Danièle Bourcier, coauteure du premier livre sur le sujet en France (Bourcier, D. \& Andel, P. V. La Sérendipité: Le hasard heureux. (Editions Hermann, 2011), on pourrait la définir comme,

l'action de tirer profit de circonstances imprévues.

La circonstance imprévue qui concerne ce billet part de la situation suivante : parti à la recherche du " Déambulatoire ", un bâtiment du campus universitaire de la Doua

(Lyon1), j'utilise la recherche image de Google qui me permet de trouver de quoi repérer le bâtiment en question :

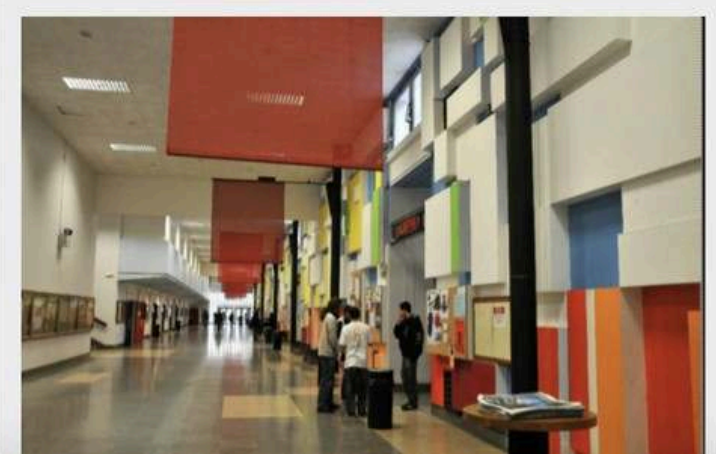

Figure 12 « Expliciter et partager l'imprévu, la sérendipité » Source : Pascal Bellanca Penel, « De la sérendipité, en particulier », Trajectoires (carnet de thèse). https://traj.hypotheses.org/208.

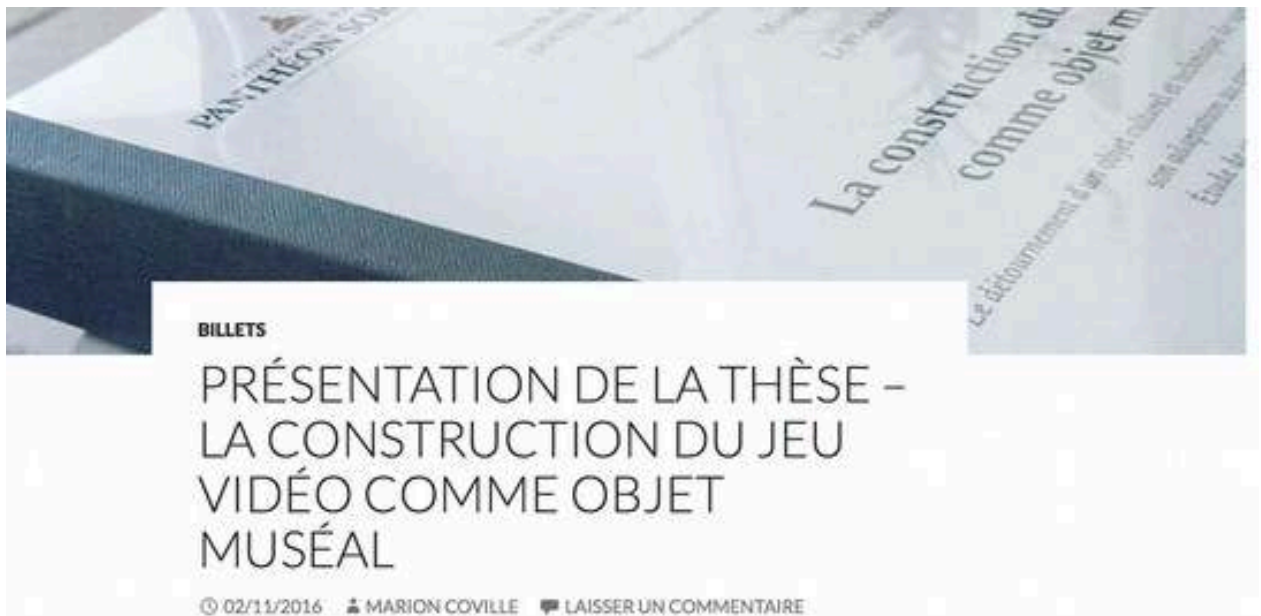

Le 19 octobre dernier, le jour de la soutenance est enfin arrivé... Le jury de soutenance était composé de Julia Bonaccorsi, Professeure des Universités - Université Lyon 2 (rapportrice et Présidente du jury), Christine Détrez, Professeure des Universités - ENS Lyon (rapportrice), Fanny Lignon, Maitresse de Conférences - Université Lyon 1 (examinatrice), Bernard Darras, Professeur des Universités Université Paris 1 Panthéon Sorbonne (examinateur) et Christophe Genin, Professeur des Universités - Université Paris 1 Panthéon Sorbonne (directeur de thèse).

Figure 13 « Présenter le sujet de la thèse » Source : Marion Coville, « Présentation de la thèse - la construction du jeu vidéo 
comme objet muséal », l'exposition en jeu (carnet de thèse). https:// expojeu.hypotheses.org/219

\section{ALA UNE, LA THËSE AU TRAVAIL, LE CHEMIN DE LONGUE ÉTUDE DU DOCTORAT \\ «C'EST PAR OÙ? » : LA CARTE IGN DU DOCTORAT. \\ (1) SEPTEMBRE 8, 2015 \& VIVIANE GRIVEAU-GENEST —LAISSERUN COMMENTAIRE}

Votre sac doctoral est prêt : vous pouvez partir... mais pas trop à l'aventure néanmoins, même si le doctorat en est une, d'aventure. Prenez le temps de regarder une carte... Sinon, vous risquez de vivre ça.

Commencez donc par a. votre Destination, « pour quoi vous le faites " (but perso, carrière universitaire, montée en compétences...) b. Vos compagnons de route: avec qui vous le faites: institutions, labo, directeur directrice de thèse. c. Sur quoi vous le faites : votre sujet.

a. Votre destination : les raisons qui font que l'un, l'une ou l'autre vont envisager un doctorat sont variées et diffèrent grandement. I est toujours utile de savoir pour vous-même dans quel but vous faites cela.

-> Vous faites cela par passion, c'est un pur hobby, vous n'en attendez que de la stimulation intellectuelle (et d'ailleurs vous gagnez très bien votre vie parce que vous travaillez dans la finance). Très bien ! Passez votre chemin, cela ne vous ennuiera aucunement de vous perdre, ce sera d'autant plus stimulant. Attention, si vous ne tra-

Figure 14 « Témoigner du parcours de la thèse "

Source : Viviane Griveau-Genest, « «C'est par où » : la carte IGN du doctorat », Voix du Livre / des livres (carnet de thèse). https://voixlivres.hypotheses.org/187.

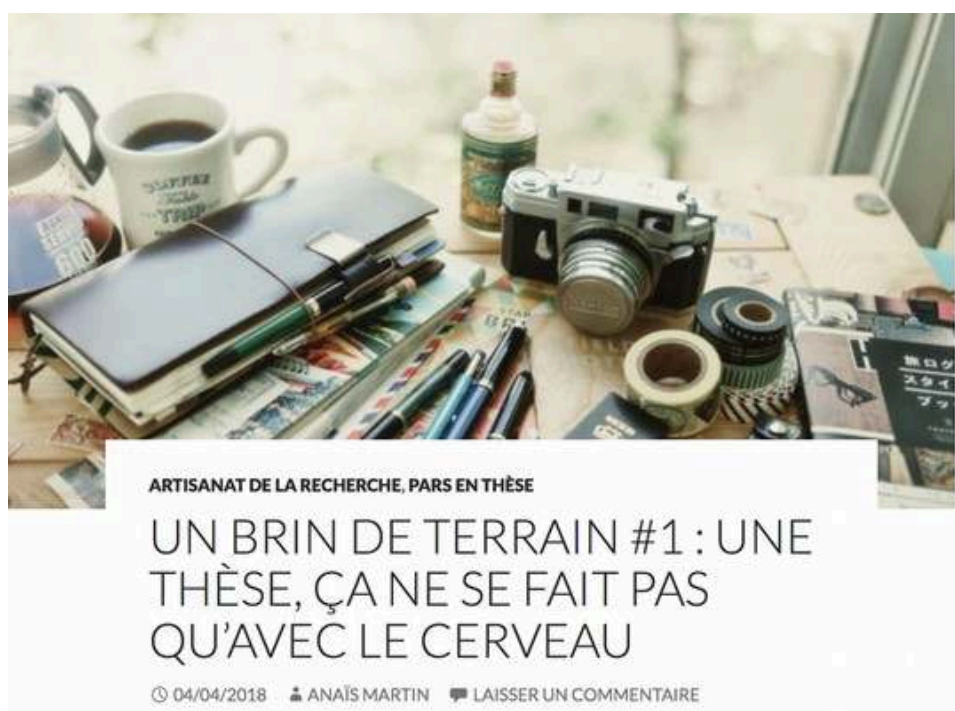

Quand je parle de mon travail autour de moi, la plupart du temps cela ne semble pas très clair pour mes interlocuteur.rice-s : « mais qu'est-ce que tu fais concrètement? * * Ça consiste en quoi faire des entretiens? \$ ${ }^{1} \mathrm{~J} a-$ vais donc envie de vous emmener avec moi sur les routes anglaises. Dans ce premier billet, je souhaite aborder les * à-côtés * de l'enquête, ces éléments que l'on ne verra pas forcément apparaître dans mes publications académiques. 
Figure 15 « Se mettre en mouvement réflexif » Source : Anaïs Martin, « Un brin de terrain \#1 : une thèse, ça ne se fait pas qu'avec le cerveau », Entre le zist et le zest (carnet de thèse). https://zistetzest.hypotheses.org/685.

\title{
Lieux de science et de recherche
}

\begin{abstract}
PAT THIERRY JOFFREDO- 13/11/2016
Il y a longtemps que j'ai envie d'écrire quelque chose sur les différents lieux, bâtiments et structures - bibliothèques, salles d'archives, centres de recherche, labos, etc. - que jai été amené à fréquenter à loccasion de mes travaux de recherche en histoire des mathématiques lors de ces dernières années. La science n'est pas qu'une activité purement intellectuelle, non géographiquement située: les lieux dans lesquels cette science se construit sont d'une importance cruciale dans ses processus d'élaboration'. A une échelle très personnelle, la fréquentation de ces lieux de science et de recherche a été un élément moteur de mon parcours d"apprenti historien. Si j'effectue l'essentiel de mon travail de recherche, d'analyse et de rédaction depuis mon bureau, chez moi, le fait de me transporter, à l'occasion d'un séjour en archives, d'un colloque ou d'une école, dans un endroit où la science s'exerce et se pratique effectivement, m'est toujours bénéfique et stimulant. Au-delà de leur fonction première ces lieux sont toujours pour moi un sujet de curiosité et d'ẻmerveillement : le prestige de bâtiments chargés d'histoire, la solennité et la quiétude des salles de lecture des archives et dés bibliothèques d'une part, et les rencontres, les échanges que l'on peut y faire d'autre part, tout cela concourt à l'immense plaisir que je ressens chaque jour à mener mes recherches, Voici une présentation * naive s; au travers de quelques exemples illustrés, d'une géographie institutionnelle personnelle, qui a toute son importance dans mon parcours au cours de ces cinq dernières années.
\end{abstract}

\section{Les archives Henri Poincaré à Nancy}

J'ai la chance d'être membre d'un laboratoire (le Laboratoire d'Histoire des Sciences et de

Figure 16 « Expliciter et partager l'ordinaire de la recherche » Source : Thierry Joffredo, "Lieux de science et de recherche ", Approches biographiques d'un texte scientifique (carnet de thèse). https://cramer.hypotheses.org/113. 
BILLETS

SOUTENANCE DE THESE
(129MARS 2017 \& MAIIEUL ROUQUETTE LAISSERUn COMmENTAIRE

Après cinq ans à Lausanne, quatre ans de recherche, un certain nombre d'heures passées à effectuer des actions parfois intéressantes (décrypter un texte, en articuler plusieurs, trouver comment automatiser toujours plus de chose avec LaTeX) parfois moins (remplir des formulaires administratifs et faire la chasse aux graphies non conventionnelles), je soutiendrai ma thèse de doctorat le 12 mai 2017 à $13 \mathrm{~h} 30$, dans la salle 319 de l'Amphipôle de l'Université de Lausanne (métro Unil-Sorge).

Ma thèse s'intitule " Étude comparée sur la construction des origines apostoliques des Églises de Crète et de Chypre à travers les figures de Tite et de Barnabé ». En voici un résumé:

La fondation d'une Église locale par un apôtre constitue un élément important dans les relations interecclésiales. Une telle fondation peut cependant être elle-même l'objet de

contestation, en particulier si le statut apostolique de la figure fondatrice n'est pas manifeste dans le Nouveau Testament. C'est pourquoi une Église peut être amenée à se construire un passé apostolique, notamment à travers la production de vies d'apôtre, lesquelles peuvent souligner tant le statut apostolique de la figure que son caractère fondateur pour l'Église.

Figure 17 « Raconter la soutenance »

Source : Maïeul Rouquette, "Soutenance de thèse ", Apocryphes (carnet de thèse). https://apocryphes.hypotheses.org/569.

DANSLES LOGES

\section{LESÉMOTIONS DE LA RECHERCHE}

(1) 07/11/2017 ¿ STÉPHANIE PIREZ-HUART - LAISSER UN COMMENTAIRE

Dimanche je donnais une conférence pour le Cercle Archéologique et Historique de Valenciennes. C'était ma première conférence longue, j'ai parlé seule plus d'une heure, puisqu'habituellement en colloque ou en journée d'études on est malheureusement limité.es. Là donc j'avais le temps. Outre ce confort (quoique relatif car il faut savoir tenir la distance, et là je dis merci l'enseignement!), cette présentation avait une saveur particulière pour moi.

Le CAHV, c'est l'une de ces sociétés savantes dont sont dotées bien des villes, grandes et petites. On y trouve de tout : des chercheur.es à la retraite, des enseignant.es du secondaire, encore en activité ou pas, des érudit.es, des amateur-rices. Mais surtout des passionné.es d'histoire, et davantage d'histoire régionale et locale. C'est un public particulier : ils/elles n'ont pas forcément tou-tes un bagage universitaire, et encore moins en histoire, donc pas forcément la méthode ou les connaissances, d'autres se sont formé-es seul-es, parfois tard. Bref c'est un public très hétérogène. De ce fait, l'exercice d'une présentation devant elles/eux c'est comme un numéro d'équilibriste : il faut fournir un travail aussi sérieux que pour un colloque bon chic bon genre tout en tenant un discours compréhensible. Faire simple sans être simpliste. Car si ce public nécessite 
Figure 18 « Expliciter et partager les émotions de la thèse »

Source : Stéphanie Pirez-Huart, « Les émotions de la recherche », Médié V@l (carnet de thèse). https://valmed.hypotheses.org/221.

\section{De mon premier colloque international}

(1) 13 juin 2017 Billets colloque, Genesis, Ma vie de doctorante

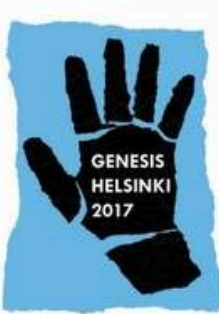

Nom: Genesis -Helsinki

Dates: du 7 au 9 juin 2017

Lieu : Helsinki, Finlande

Objet: premier colloque international au-

tour de la génétique, au sens large du

terme.

Genesis Helsinki : présentation Le colloque Genesis Helsinki était le premier du nom : c'est la première fois que des chercheurs se réunissaient pour partager à propos de génétique. Entendez là génétique au sens large du terme : il s'agissait de génétique textuelle bien sûr, mais aussi de génétique de l'image, de la musique, du théâtre, du cinéma. 54 communicants se sont succédés tout au long de ces 3 journées.

Pour ma part, je communiquais sur mes recherches, certes débutantes, mais pour montrer qu'il existe un corpus en génétique de l'écriture pour la jeunesse.

La Finlande : un attachement particulier Faire cette communication en Finlande avait un sens tout particulier pour moi : la Finlande, je la connais bien pour y avoir étudié comme étudiante Eramus, il y a quinze ans. C'était pour ma dernière année d'études (à

Figure 19 «Communiquer sur ses activités »

Source : Solène Audebert-Poulet, « De mon premier colloque international ", Jeunesses d'albums (carnet de thèse). https://albumgenetic.hypotheses.org/180. 


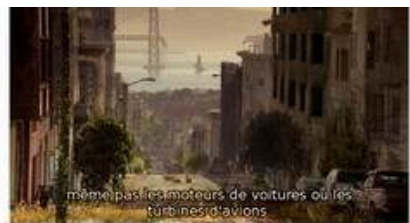

\title{
" Révolution ", la dernière série télé américaine réactionnaire
}

\begin{abstract}
Aussi louable que soit la capacité de digestion de l'industrie hollywoodienne des thèmes les plus actuels de notre société (et en particulier des épisodes de guerre, depuis Apocalypse Now en 1979 jusqu’à dans la vallée d'Elah en 2007 sur la guerre en Irak), cette retranscription fictionnelle de l'actualité la plus brulante n'apporte pas toujours un regard critique sur la société. Bien au contraire.

Dernier exemple en date, la série Révolution (diffusée sur NBC depuis le 17 septembre 2012 et produite entre autres par J.J. Abrams) suit les aventures d'une famille quinze ans après le " black out ", c'est-à-dire la fin de l'électricité, et recycle ainsi les peurs contemporaines portant sur la fin des énergies fossiles faciles et l'épuisement des ressources. Qu'en est-il
\end{abstract}

Figure 20 « S'intéresser à un sujet populaire »

Source : Daphné Vialan, « « Révolution », la dernière série télé américaine réactionnaire ", L'habiter environnemental (carnet de thèse). https://

habiter.hypotheses.org/75.

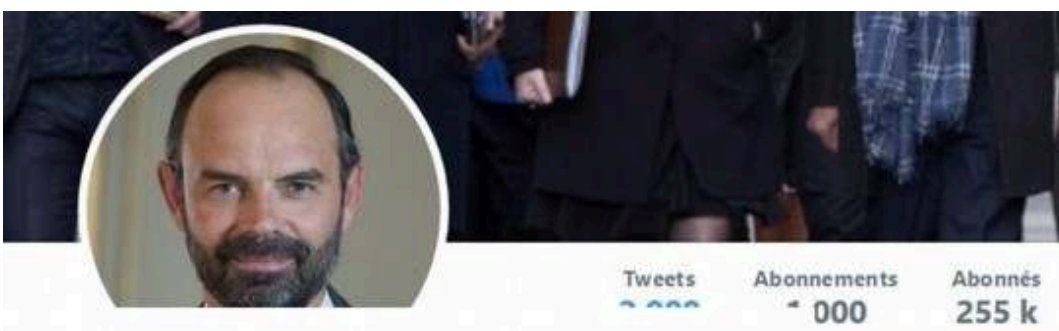

C'EST PAS QUE D'LA COM I, COMMUNICATION DE L'ETAT

C'EST PAS QUE D'LA COM! L'INSULTE ET LAMOQUERIE SUR TWITTER

Q 17/01/2018 \& MATHIEUFUSI - LAISSERUNCOMMENTAIRE

Dans une petite étude faite maison, publiée récemment sur ce carnet, nous avons relevé que les activités de communication du premier ministre Édouard Philippe subissait moquerie et insulte. Plus généralement, ses tweets s'inscrivent dans une pratique critique de la part des usagers de Twitter qui profitent du réseau social numérique pour attaquer l'action du gouvernement. Nous présentons ici des éléments de réflexion qui approfondissent à la fois notre idée du - c'est pas d'la com ! * et le questionnement de la communication de l'́tat sur les réseaux sociaux numériques.

Critique de l'apparence : intérêt paradoxal mais 
Figure 21 « Mettre son expertise en jeu »

Source : Mathieu Fusi, « C'est pas que d'la com ! - L'insulte et la moquerie sur Twitter », Point-virgule (carnet de thèse). https://ptvirgule.hypotheses.org/310.

\section{Qu'est-ce qu'une langue en danger?}

PAR JHONNATAN RANGEL. PUBLLÊ 15/11/2015-MIS Á JOUR 30/06/2017

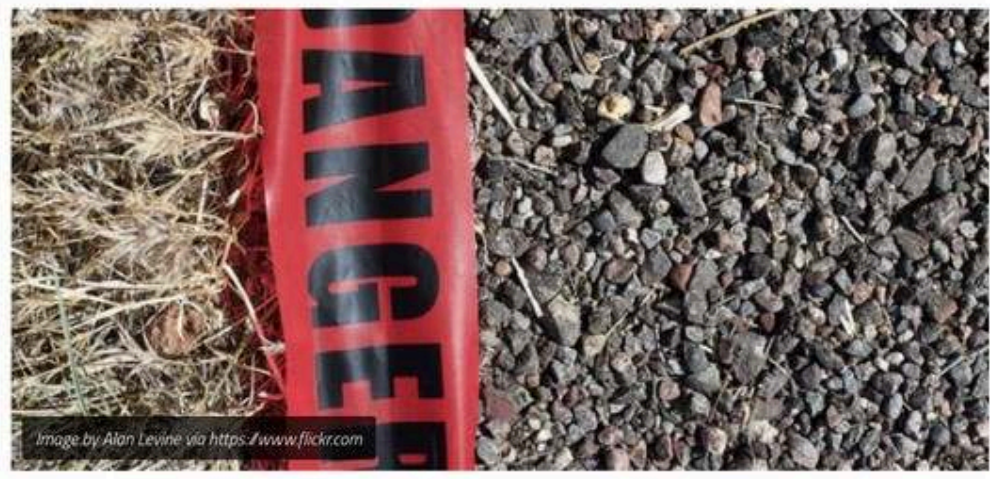

En 1992, Michael Krauss a lancé un cri d'alarme à la communauté internationale lorsquil affirmait que la moitié des 6000 langues parlées dans le monde pourrait disparaître avant la fin de ce siècle. Ce cri d'alarme, comme on dit en anglais, went viral, dans le monde de la recherche ainsi que dans le grand public. Et lorsqu'on parle des langues en danger, on est obligé d'en faire référence.

Mais, en gros, qu'est-ce que cela veut dire qu'une langue va ả disparaitre ? Krauss, en s'inspirant du domaine de la biologie, a comparé les langues en danger aux espèces biologiques en voie d'extinction. Dans les deux cas, le nombre « d'espèces » (locuteurs) et le taux de « reproduction "

Figure 22 « Partager des questionnements et non des certitudes » Source : Jhonnatan Rangel, «Qu'est-ce qu'une langue en danger? », Where's the last speaker (carnet de thèse). https://wils.hypotheses.org/117. 


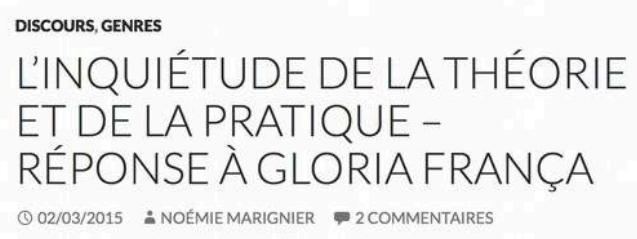

Ce billet est une réponse à celui de Gloria França que vous pouvez lire sur son carnet Ces mots qui voyagent.

Lorsque, la semaine dernière, au cours d'une discussion sur la difficulté à concilier théorie et pratique, Gloria m'a lu ces quelques lignes de son petit carnet, j'ai éprouvé ce joyeux sentiment de proximité et d'entente dans une amitié naissante. Gloria écrit dans son billet à propos de nos discussions " c'est une belle rencontre que la nôtre ", et c'est aussi ce que je ressens : le partage de mêmes interrogations, de mêmes problèmes et surtout de mêmes inquiétudes - le mot est loin d'être choisi au hasard - et je pense que c'est ce dernier point qui nous a donné envie d'écrire ces billets jumeaux.

Gloria avait donc écrit sur son petit carnet :

Mon rapport avec la théorie et la praxis féministe : dans mon cas c'est la théorie qui est venue d'abord - et avec elle, par la suite, une praxis politique issue de la théorie, mais pas uniquement. Et ces deux facettes restent distincts mais imbriquées. Avant d'avoir accès à la théorie ce que je ressentais c'était l'expérience de l'oppression, cependant je ne la formulais pas.

Figure 23 " Entrer en conversation scientifique » Source : Noémie Marignier, «L'inquiétude de la théorie et de la pratique - Réponse à Gloria França », Corps et mots (carnet de thèse). https://corpsmots.hypotheses.org/234.

\section{DIVERS}

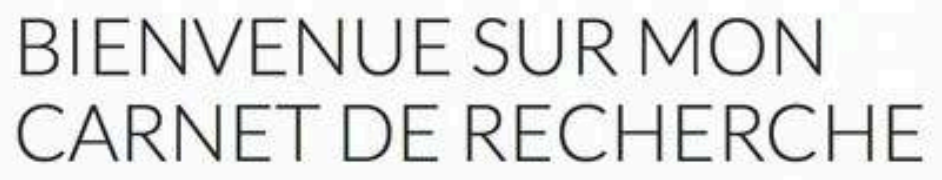

(1) 19 OCTOBRE 2011 ¿ KEVINDURUISSEAU LAISSER UNCOMMENTAIRE

Ce carnet vise à vous présenter les avancées de ma thèse qui s'intitule : "Nouvelle géographie et territorialisation des énergies renouvelables. L'exemple du solaire photovoltaïque dans le Sud de la France : acteurs, facteurs et jeux d'échelles«. Par ailleurs, il sera le lieu d'une réflexion conceptuelle et notionnelle concernant la transition énergétique, le développement durable, la territorialisation des politiques publiques, etc. Enfin, ce carnet entend suivre l'actualité française et européenne en matière énergétique.

Figure 24 « Souhaiter la bienvenue » Source : Kévin Duruisseau, « Bienvenue sur mon carnet de recherche », Une géographie de l'énergie (carnet de thèse). https://nrgrenouv.hypotheses.org/41. 
Aux lectrices et lecteurs des Inrockuptibles de 1986 à 1998

3 réponses

Vous avez été lectrice ou lecteur des Inrockuptibles entre 1986 et 1998. J'ai besoin de votre aide, de votre contribution.

Le questionnaire ci-dessous prends moins de 5 minutes à remplir et me permettra de cerner un peu mieux la réception du magazine par son lectorat au cours de la période que j'étudie. Plus les réponses seront nombreuses et plus elles seront intéressantes à exploiter.

A vous souris et vos souvenirs ! Et merci aussi !

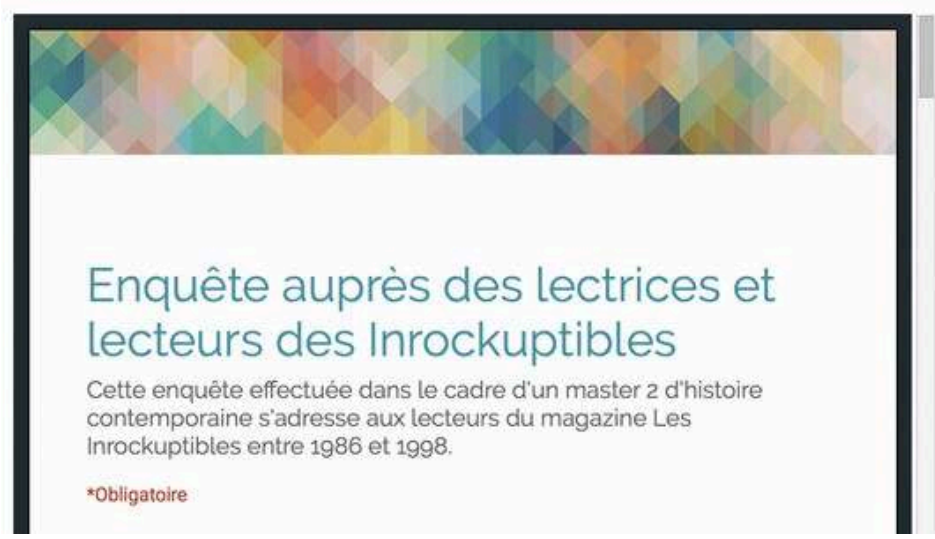

Figure 25 « Appeler à la participation »

Source : Véronique Servat, « Aux lectrices et lect1eurs des Inrockuptibles de 1986 à 1998 », La Factory (carnet de thèse). https://lafactory.hypotheses.org/112.

\section{Écrire pour le « grand public »}
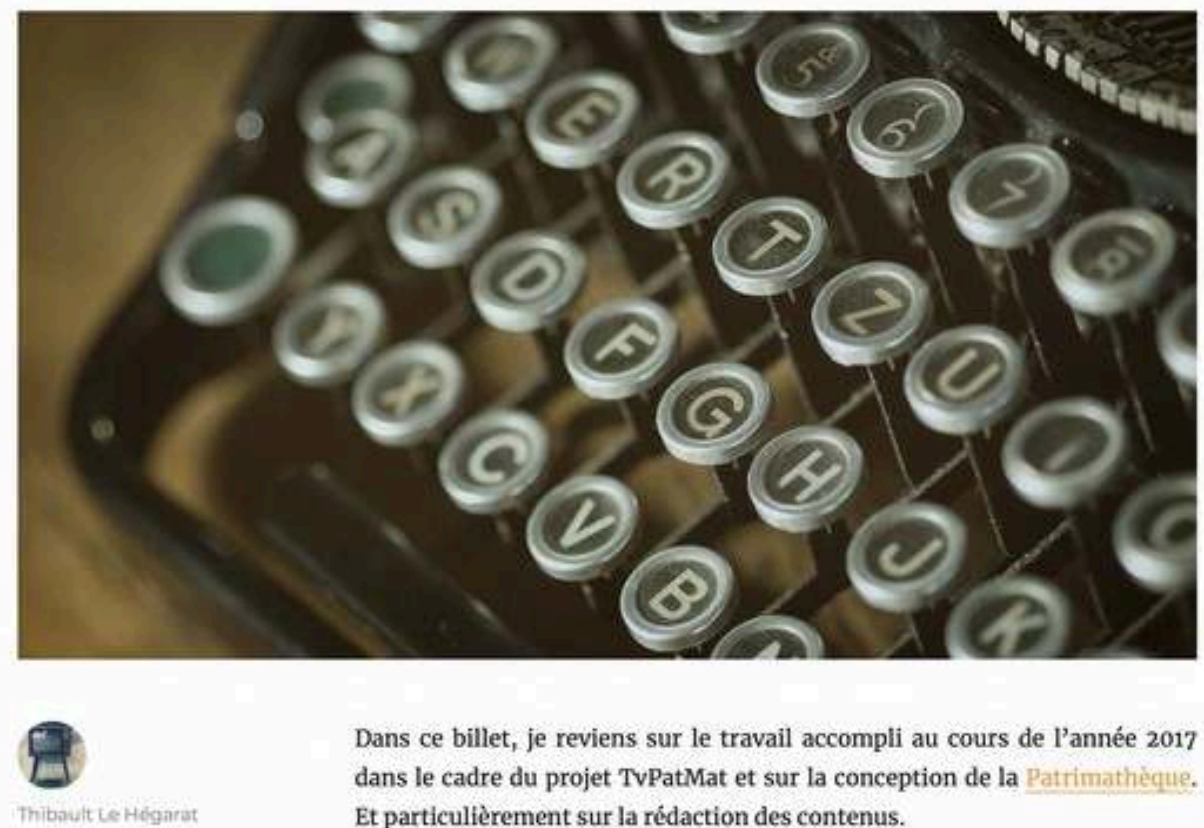

Dans ce billet, je reviens sur le travail accompli au cours de l'année 2017 dans le cadre du projet TvPatMat et sur la conception de la Patrimathèque. zano/2017 Et particulièrement sur la rédaction des contenus. 
Figure 26 « Ecrire pour le grand public » Source : Thibault Le Hégarat, « Ecrire pour le « grand public » », Patrimoine et télévision (carnet de thèse). https://tvpatri.hypotheses.org/992.

\title{
Ecriture et lecture comme exercices spirituels : retour sur 5 années de blogging scientifique
}

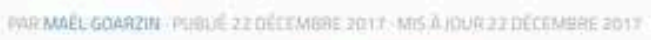

Ginq annèes ont passé depuis man premier billet sur ce carnet de recherche ll est temps de faire le point sur cette pratique d'écriture et de lecture qui mia accompagné tout au long de mon parcours doctoral D'abord conçu comme moven de prèsenter mes recherches sur le mode de vie néoplatonicien (ẩ travers la catégorie Ma thèse), ce carnet est rapidement devenu un outil beaucoup plus large, me permettant de réflëchir non seulement aux giratiques de recherche et décriture propires au doctorat lâ travers la catégorie Ma vie de cloctorant), mais aussı à la pertinence et à Yliniactualité des textes antiques : que peut nous apporter, anjourdhui, la lecture des philosophes antiques? En quoi la philosophe antique peut-elle ềtre utile pour une rẻflexion sur notre maniêre de vivre au quotidien?

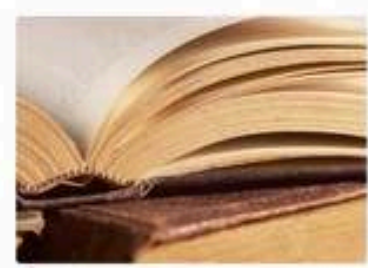

Je me suis très vite aperçu que ce camet était le lieu idéal pour toucher un public plus large que ie public universitaire, et cest pourquoi i'ai toujours recherchẻ la clartế du propos tout en restant précis dans línterpretation des textes et dans les commentaires proposés. Lieu décriture privilégiè, v compris dans les moments plus difficiles du partours doctoral, ce camet de recherche reflète un certain nombre de lectures faites au cours des demières annees, La lecture et récriture parfois aride des articles scientifiques ont laissé leur place à un type d'ècriture beaucoup plus léger et fluide ; la lecture des textes antiques est devenue oautant plus irtéressante que les réflexions suscitées pouvaient être partagées sur ce carnet; finalement, lecture et écriture sant deveris pour mơi des pratiques quotidiennes, et lintérêt suscité par ces pratiques a été décuplé par l'activité de blogging scientifique.
\end{abstract}

Figure 27 « Pourquoi blogguer?»

Source : Maël Goarzin, « Ecriture et lecture comme exercices spirituels : retour sur 5 années de blogging scientifique ", Comment vivre au quotidien? (carnet de thèse). https:// biospraktikos.hypotheses.org/3281.

\section{NOTES}

1. Source : rapport d'activités 2017 d'OpenEdition.

2. Voici ces types de carnet accompagnés chaque fois, entre parenthèse, du nombre de carnets au catalogue le 10 septembre 2018 : Carnet de programme de recherche (669), Carnet de chercheur (427) ; Carnet de structure de recherche (389) ; Carnet de séminaire (227) ; Carnet de thèse (175) ; Carnet d'événement (98) ; Carnet de veille (98) ; Carnet de vulgarisation (83) ; Carnet de master (76) ; Carnet d'accompagnement de publication (65) ; Carnet de terrain (64) ; Carnet de débat (62) ; Carnet de méthodologie (56) ; Carnet de bibliothèque (50) ; Carnet média (11) ; Bibliographie (2) ; Carnet de réseau (1)

3. Faury et Giraud (13 février 2012) "Le carnet de thèse". Espaces réflexifs [carnet de recherche]. http://reflexivites.hypotheses.org/641

4. Groupe d'Intérêt Scientifique, Unité Régionale de Formation à l'Information Scientifique et Technique.

5. Ces entretiens permettent de mettre en perspective les analyses que nous faisons en tant que chercheure.es sur les carnets Hypotheses.org avec ce que les carnetier.es disent qu'elles et ils font et avec le sens explicite qu'elles et ils donnent à leur pratique.

6. Selon une démarche proche de celle développée dans le cadre de ma thèse, associant à la récolte de traces écrites - en l'occurrence les billets - le commentaire des pratiques de communication par les auteurs eux-mêmes. 
7. Et non pas le carnet de recherche dans sa globalité et en lien avec la logique dans laquelle il s'inscrit, comme nous avons pu le faire précédemment avec Elsa Poupardin dans le cadre du projet HYCAR - article à paraître.

8. En ayant conscience des divergences par rapport à cette schématisation selon les sujets de recherche, les terrains, les disciplines, les laboratoires, etc.

9. Ce type d'appropriation de "l'espace du carnet de recherche» me paraît de plus en plus fréquent sur Hypotheses.org, peut-être à l'exception des carnets de doctorant.es et de chercheur.es. Il nous faudra confirmer ou infirmer cette hypothèse d'un point de vue quantitatif dans le cadre du projet HYCAR.

10. Je rejoins Joëlle Le Marec dans son intérêt pour les composites et les pratiques de communication dans l'activité de recherche, et en l'occurrence concernant les doctorant.e.s : «C'est mon ambition ici : déplacer autant que faire se peut le point aveugle entre des pratiques de communication habituellement laissées hors champ scientifique mais nécessaires à la pratique de recherche et les pratiques explicites normées dont on fait état publiquement pour rendre compte de ce que sa pratique a de scientifique, pour la faire reconnaître comme telle, et pour intéresser les personnes avec qui on partage la volonté de scientificité " (Le Marec, 2002, habilitation à diriger des recherches). J'entre aussi en résonance avec les travaux de Marie-Anne Paveau (2010) sur le désir épistémologique et de Baudouin Jurdant sur le désir de scientificité (1999).

11. Inspirée en science de l'information et de la communication par les travaux de Joëlle Le Marec sur les situations de communication, et par l'épistémologie féministe et la standpoint epistemology, des travaux de Sandra Harding, Donna Haraway et Maria Puig de la Bellacasa, on pourrait parler également de savoirs « de subalternes ».

12. Terme utilisé par l'organisation ATD Quart Monde dans sa démarche de croisement des savoirs : https://www.atd-quartmonde.fr/sengager/dans-votre-milieu-professionnel/ croisementdessavoirs/le-croisement-des-savoirs-et-des-pratiques/

13. Les figures permettant de donner un exemple pour chaque type de billet identifié sont présentées en annexe.

14. Exemples de billets de ce type $: 1 /$ Partage de lectures :

Claire-Lise Gaillard, " [Lecture] Revue Sensibilités n 3 - « Corps au paroxysme » ». Le marché de la rencontre 1850 -1950, https://marcherenc.hypotheses.org/691.

Dimitri Julien, «Retour sur Ivan Jablonka, Histoire des grands-parents que je n'ai pas eus ». Écritures historiques (carnet de thèse). https://ecrirehist.hypotheses.org/132.

Jhonnatan Rangel, « Mes lectures pour les vacances d'été », Where's the last speaker? (carnet de thèse). https://wils.hypotheses.org/201.

2/ Partages de bibliographies :

Lola Druilhe, "Aumisme : Une bibliographie «sciences religieuses" ». Enième ère (carnet de thèse). https://nmr.hypotheses.org/231.

Rosemonde Letricot, «Tour d'horizon des éditions numériques de sources ». Prosopoly (carnet de thèse). https://prosopoly.hypotheses.org/134.

Anaïs Martin, « Mes plus beaux récits de terrain : liste évolutive, subjective et non-exhaustive ». Entre le zist et le zest (carnet de thèse). https://zistetzest.hypotheses.org/321.

15. Exemples de billets de ce type :

Jeannier, Fabien. "De l'intérêt d'être sur place ». Usages des langues vivantes (carnet de thèse), https://languesvivantes.hypotheses.org/218.

Martin Lafréchoux, "L'éternel retour du meme », Le texte en miettes (carnet de thèse). https:// miettes.hypotheses.org/181.

Stéphanie Messal, «[Explorer l'Urbex] La génèse ». Misanthropologue (carnet de thèse). https:// misanthropologue.hypotheses.org/1511.

Caroline Muller, «Un château d'enfance ». Acquis de conscience (carnet de thèse), https:// consciences.hypotheses.org/1290. 
Jhonnatan Rangel, «Le travail de terrain avec les «derniers locuteurs » du zoque ayapaneco (Mexique)». Where's the last speaker? (carnet de thèse). https://wils.hypotheses.org/80.

SLJ, « Autour de la thèse // Sur les traces des étudiants indiens à Londres (1)». Voies étudiantes (carnet de thèse). https://etudiants.hypotheses.org/361.

16. Exemples de billets de ce type :

Caroline Muller, "Cinq ans d'usage de Zotero, un bilan ». Acquis de conscience (carnet de thèse). https://consciences.hypotheses.org/1184.

Daniel Pélissier, "Quelle identité numérique pour un doctorant?». Présence numérique des organisations (carnet de thèse). https://presnumorg.hypotheses.org/318.

Damien Petermann, «Doctorant et liseuse électronique : un retour d'expérience (1re partie)». L'image de Lyon (carnet de thèse). https://imagelyon.hypotheses.org/813.

Stéphanie Pirez-Huart, « Du bon usage des outils numériques et de la courtoisie dans une thèse ». Médié V@l (carnet de thèse). https://valmed.hypotheses.org/307.

Ophélie Siméon, « Ressources pour la présentation des références bibliographiques ». Usages des langues vivantes (carnet de thèse). https://languesvivantes.hypotheses.org/195.

17. Exemples de billets de ce type :

Caroline Corvasce, "Différence entre savoir et connaissance». Formation passion (carnet de thèse). https://formation.hypotheses.org/250.

Eglantine, "Quelques enjeux épistémologiques de l'analyse du web et des big data ». La ruée vers la donnée (carnet de thèse). https://bigdata.hypotheses.org/81.

Sébastien Marlair, «Histoire et scientificité ». Postures \& récits (carnet de thèse). https:// postures.hypotheses.org/292.

Sabine Rabourdin, "La science et ses valeurs: enjeu d'émancipation dans l'Inde moderne ». L'Indécise (carnet de thèse). https://indecise.hypotheses.org/489.

Jhonnatan Rangel, «Toutes les langues dans ma bouche ». Where's the last speaker? (carnet de thèse). https://wils.hypotheses.org/477.

Sandrine Teixido, « Musiques du monde : premières réflexions sur une catégorie qui n'existe pas \# 1 ». Agir en Musiques (carnet de thèse). https://musicagir.hypotheses.org/17.

18. Exemples de billets de ce type :

Pascal Bellanca-Penel, « De la sérendipité, en particulier. ». Trajectoires (carnet de thèse). https:// traj.hypotheses.org/208

Mathilde Christmann, «Douter...». Partitions (carnet de thèse). https:// partitions.hypotheses.org/179.

Marc Lenormand, "Comment ne pas mener un programme d'entretiens ». Usages des langues vivantes (carnet de thèse), https://languesvivantes.hypotheses.org/179.

19. Exemples de billets de ce type :

L'équipe ACT, «Comment devient-on chercheur en sciences sociales? (2014-2015) ». Les aspects concrets de la thèse (carnet de thèse). https://act.hypotheses.org/4514

$\mathrm{MD}$, « Face à la crise des financements doctoraux, savoir affirmer la valeur de sa recherche ». Les aspects concrets de la thèse (carnet de thèse). https://act.hypotheses.org/3414.

Sandrine Teixido, "Position du chercheur et ethnographie multi-située ». Agir en Musiques (carnet de thèse). https://musicagir.hypotheses.org/46.

20. Exemples de billets de ce type :

Marion Coville. " Présentation de la thèse - La construction du jeu vidéo comme objet muséal ", L'expo en jeu (carnet de thèse). https://expojeu.hypotheses.org/219.

Viviane Griveau-Genest, "“C'est par où ?" : la carte ign du doctorat. ", Voix du Livre / des livres. (carnet de thèse). https://voixlivres.hypotheses.org/187.

Thierry Joffredo, "Lieux de science et de recherche", Approches biographiques d'un texte scientifique (carnet de thèse). https://cramer.hypotheses.org/113. 
Anaïs Martin, "Un brin de terrain \#1: Une thèse, ça ne se fait pas qu'avec le cerveau », Entre le zist et le zest (carnet de thèse). https://zistetzest.hypotheses.org/685.

Maïeul Rouquette, "Apocryphes | Définitions, statuts, usages, productions ", Apocryphes (carnet de thèse). https://apocryphes.hypotheses.org/.

21. Exemples de billets de ce type :

Charles Bonnot, " «You've got some nerve asking me a question like that! » ", Funny you should say that (carnet de thèse). https://docnroll.hypotheses.org/119.

Eglantine, «Clarification un peu irritée sur ce qu'est et n'est pas un algorithme ", La ruée vers la donnée (carnet de thèse). https://bigdata.hypotheses.org/59.

Claire-Lise Gaillard, "Qu'est-ce que l'intime en sciences sociales? Réflexions théoriques ", Le marché de la rencontre 1850 -1950 (carnet de thèse). https://marcherenc.hypotheses.org/700.

Sébastien Marlair, « De la science à l'affect, de la politique à l'esthétique », Postures \& récits (carnet de thèse). https://postures.hypotheses.org/264.

Stéphanie Pirez-Huart, "Les émotions de la recherche ", Médié V@l (carnet de thèse). https:// valmed.hypotheses.org/221.

Marine Rouch, "Emotions de l'historien.ne et archives de l'intime ", Chère Simone de Beauvoir (carnet de thèse). https://lirecrire.hypotheses.org/597.

22. Je fais notamment référence ici à l'épistémologie du lien proposée par Florence Piron comme alternative à l'épistémologie positiviste (Piron, 2017).

23. Exemples de billets de ce type :

Pascal Bellanca-Penel, «Projet Doctoral (Janvier 2013)», Trajectoires (carnet de thèse). https:// traj.hypotheses.org/33

Gaëlle Debeaux, "Thèse en ligne", Multiplication des récits (carnet de thèse). https:// multirecits.hypotheses.org/321.

Kevin Duruisseau, «Mon projet de recherche», Une géographie de l'énergie (carnet de thèse). https://nrgrenouv.hypotheses.org/45.

Céline LeGall, «Publication en ligne de ma thèse : «Giovanni Poleni (1683-1761) et l'Académie Royale des sciences de Paris » en mars 2018. ", Autour de Poleni (carnet de thèse). https:// poleni.hypotheses.org/461.

Anaïs Martin, "Ma première communication. Entrée dans la cour des jeunes chercheur·e's ", Entre le zist et le zest (carnet de thèse). https://zistetzest.hypotheses.org/57.

24. Exemples de billets de ce type :

1/ Problématiser l'actuel, le quotidien et le populaire

Nada Chaar, "Pourquoi les profs râlent tout le temps ?", Enseigner dans le secondaire (carnet de thèse). https://enseigner.hypotheses.org/312.

Maël Goarzin, «Le choix de vie dans les séries TV », Comment vivre au quotidien ? (carnet de thèse). https://biospraktikos.hypotheses.org/2969.

Christophe Meunier, "Habiter le monde selon Ikéa ", Les territoires de l'album (carnet de thèse). https://lta.hypotheses.org/861.

2/ Avis d'expert

Line Domingues, « Devoirs à la maison : leur utilité ? (1/2)», Identité - altérité (carnet de thèse). https://idal.hypotheses.org/167.

Alessia Smaniotto, «Le Rapport Entre Journalistes et Vérité Comme Problème Public », Doveritas (carnet de thèse). https://doveritas.hypotheses.org/44.

Alexis Yannopoulos, «Imposture intellectuelle ou naïveté innocente ?», Les fruits du magnolia (carnet de thèse). https://estraven.hypotheses.org/84.

25. Exemples de billets :

Fleur Chabaille-Wang (王铚花), «Pourquoi l'histoire de la concession française de Tianjin? Réflexions "existentielles" au fil de la thèse... », Tianjin Spatial History (carnet de thèse), https:// tsh.hypotheses.org/333. 
Mathieu Fusi, «Pourquoi la définition du concept de communication est une posture épistémologique?», Critiquables (carnet de thèse). https://critiquabl.hypotheses.org/17. Viviane Griveau-Genest, «Style cliché ou originalité : réflexions autour d'un problème.», Voix du Livre / des livres (carnet de thèse). https://voixlivres.hypotheses.org/180.

26. Exemples de billets de ce type :

Noémie Marignier, "L'inquiétude de la théorie et de la pratique - Réponse à Gloria França ", Corps et Mots (carnet de thèse). https://corpsmots.hypotheses.org/234.

Louis Morelle, «Lettre à un ami spinoziste», Marginalia (carnet de thèse). https:// henkaipan.hypotheses.org/54.

27. Témoignages recueillis en entretiens.

28. Les travaux de Pierre Mercklé (Hyposphère, première version: http://pierremerckle.fr/ 2011/06/lhyposphere/) et Josquin Debaz (Hypothèsosphère, première version: https:// socioargu.hypotheses.org/1921), présentés le 8 janvier 2019, permettent de rendre compte de manière précise et réactualisée des différents types de liens construits entre les carnets, via les auteur.es eux-mêmes ou via les trajectoires de lectures des visiteurs.

29. C'est-à-dire à l'ouverture de leur carnet et dans les premiers temps de la « vie » de leur carnet de thèse.

30. Les doctorant.es peuvent appréhender la lecture effective de leurs billets et le type de lectures par les statistiques fournies par leur carnet, qui leur donnent à la fois des informations quantitatives et qualitatives.

31. Exemples de billets de ce type :

Fleur Chabaille-Wang (王钰花), 《蛇年吉祥! Joyeuse année du serpent! », Tianjin Spatial History (carnet de thèse), https://tsh.hypotheses.org/376.

Mathilde Christmann, "*Bonne année 2015*", Partitions (carnet de thèse). https:// partitions.hypotheses.org/191.

Caroline Corvasce, «Bilan 2014 du blog : merci !», Formation passion (carnet de thèse). https:// formation.hypotheses.org/260.

Kevin Duruisseau, «Bienvenue sur mon carnet de recherche », Une géographie de l'énergie (carnet de thèse). https://nrgrenouv.hypotheses.org/41.

Benoît Kermoal, "Je ne vous ai pas trop ennuyé avec mes salades ? ", Enklask / Enquête (carnet de thèse). https://enklask.hypotheses.org/984.

Véronique Servat, "Aux lectrices et lecteurs des Inrockuptibles de 1986 à 1998 ", La Factory (carnet de thèse). https://lafactory.hypotheses.org/112.

Alexis Yannopoulos, "reprise des activités », Les fruits du magnolia (carnet de thèse). https:// estraven.hypotheses.org/72.

32. Suivant Marielle Macé (2016), au sens d'une manière de défendre un rapport au monde, en l'occurrence au monde de la recherche et de la science.

\section{ABSTRACTS}

Les blogs de doctorant.es appelés sur la plateforme Hypothèses « carnets de thèse » font émerger de nouvelles formes de vulgarisation par la tension que la pratique du blog scientifique exerce entre logique de partage et logique de publication; entre écritures ordinaires et finaliséesnormées; entre subjectivité, objectivité et scientificité; entre oralité et scripturalité ; entre diffusion ou ouverture des savoirs. Appuyés sur des exemples concrets cet article s'intéresse aux 
effets d'une telle vulgarisation, en particulier en termes de réflexivités.

Dans leurs carnets de recherche, les doctorant.es gardent en effet la trace de l'expérience vécue de la thèse, inscrivent un rapport à leur sujet, à leur terrain, à leurs objets, à leurs rencontres, à leurs lectures, etc. Elles et ils donnent de la place à l'incertitude, aux processus de construction des connaissances, des méthodes, aux questions épistémologiques. Et lorsque les doctorant.es parlent en leur nom, elles et ils contribuent à de nouvelles formes de narration et d'incarnation de la recherche, à la première personne du singulier. Les carnets de thèse hébergent ainsi de nouvelles formes de communication sur la science, où il s'agit de parler (de) la science et de parler (de) sa thèse de manière située et en rupture avec une représentation positiviste de la science et de l'activité de recherche.

\section{INDEX}

Mots-clés: énonciation, récit, réflexivité, expérience, numérique, vulgarisation, médiation, carnet de recherche, blog

\section{AUTHOR}

\section{MÉLODIE FAURY}

Mélodie Faury est docteure en sciences de l'information et de la communication dans le champ des études de sciences. Elle enseigne en tant que PRAG les sciences-sociétés et les enjeux des sciences ouvertes à l'Université de Strasbourg. Chercheuse associée au LISEC (Laboratoire Interuniversitaire des sciences de l'éducation et de la communication), équipe ATIP (Activité, travail, identité professionnelle), elle travaille sur les logiques de communication dans les pratiques de recherche, la réflexivité et les expérimentations épistémologiques. 